\title{
eRHIC ERL modeling in Zgoubi
}

\author{
F. Meot, S. Brooks, Y. Hao, Y. Jing, \\ V. Ptitsyn, D. Trbojevic, N. Tsoupas
}

\section{Collider-Accelerator Department Brookhaven National Laboratory Upton, NY 11973}

\author{
U.S. Department of Energy \\ Office of Science, Office of Nuclear Physics
}

Notice: This document has been authorized by employees of Brookhaven Science Associates, LLC under Contract No. DE-SC0012704 with the U.S. Department of Energy. The United States Government retains a nonexclusive, paid-up, irrevocable, world-wide license to publish or reproduce the published form of this document, or allow others to do so, for United States Government purposes. 


\title{
eRHIC ERL modeling in Zgoubi
}

\author{
F. M’eot, S. Brooks, Y. Hao, Y. Jing, V. Ptitsyn, D. Trbojevic, N. Tsoupas \\ BNL C-AD, Upton, LI, NY 11973 \\ January 2016
}

\begin{abstract}
This Note discusses on-going work regarding the modeling of eRHIC ERL in the ray-tracing code Zgoubi. The various pieces of the recirculator puzzle, their optical properties and their assemblage into an operational input data file in are addressed. The Note reports in particular on preparatory stages toward extensive end-to-end 6D polarized electron bunch transport simulations, which yield methods, as well a series of preliminary qualitative outcomes, discussed as well.
\end{abstract}

BNL/C-AD Note C-A/eRHIC/49 


\section{Contents}

1 Introduction $\quad 3$

2 FFAG2 recirculation loop $\quad \mathbf{6}$

2.1 Structure of the FFAG2 loop . . . . . . . . . . . . . . . . . . . . . 6

2.2 Characteristics of the arc cell . . . . . . . . . . . . . . . . . . 7

2.3 Synchrotron radiation effects, turn-by-turn f . . . . . . . . . . . . . . . 7

2.3.1 Working hypotheses . . . . . . . . . . . . . . . . . . 7

2.3 .2 Orbit outcomes . . . . . . . . . . . . . . . . . . . . 7

2.3 .3 SR $\operatorname{loss} \ldots \ldots \ldots \ldots \ldots \ldots \ldots \ldots$

2.4 23-loop up-down cycle in a simplified, 6 -arc ring . . . . . . . . . . . . . . . . . . 10

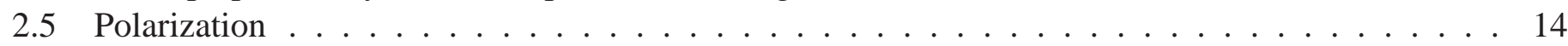

2.5.1 Synchrotron radiation effects, turn-by-turn f . . . . . . . . . . . . . . 14

$2.5 .2 \quad$ 23-loop up-down cycle in a 6 -arc ring . . . . . . . . . . . . . . . . . 14

3 Three more pieces $\quad 10$

$3.1 \operatorname{Linac} \ldots \ldots \ldots \ldots \ldots \ldots \ldots \ldots \ldots$

3.1 Chambers matrices . . . . . . . . . . . . . . . . . . . 16

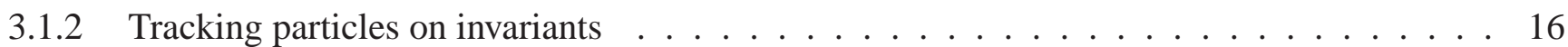

3.1 .3 Bunch transport . . . . . . . . . . . . . . . . . . . . . . 16

3.2 Spreader and merger sections f . . . . . . . . . . . . . . . . . . . 18

4 ERL optics, complete $r$

5 Tracking the ERL $\quad \mathbf{2 2}$

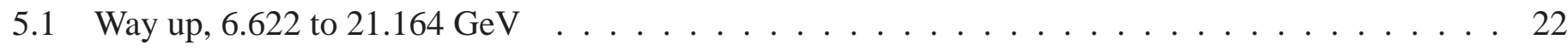

$5.1 .1 \quad$ Beam ellipses at linac ends . . . . . . . . . . . . . . . . . . . 22

5.1 .25000 -particle bunches at linac ends . . . . . . . . . . . . . . . 22

5.2 Up-down cycle in the FFAG2 stage ERL . . . . . . . . . . . . . . . . . . . . . . . . . . . . . . . . . . . . . . .

$5.2 .1 \quad$ Linac damping off and SR off . . . . . . . . . . . . . . . . . 25

5.2 .2 Linac damping and SR set . . . . . . . . . . . . . . . . . . 25

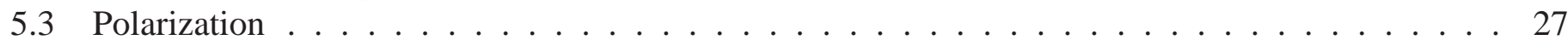

$\begin{array}{llr}6 & \text { Prospects } & 28\end{array}$

$\begin{array}{lr}\text { Appendix } & 29\end{array}$

$\begin{array}{lrr}\text { A FFAG2 arc cell in Zgoubi } & 29\end{array}$

$\begin{array}{llr}\text { B A linac cavity in Zgoubi } & 29\end{array}$

\begin{tabular}{ll} 
References & 30 \\
\hline 0
\end{tabular} 


\section{Introduction}

This Note discusses on-going work regarding the modeling of eRHIC ERL [1] in Zgoubi [2, 3]. The various pieces of the recirculator puzzle, their optical properties and their assemblage in an operational input data file, toward extensive electron beam dynamics and polarization simulations, are addressed. These eRHIC model developments at the present stage yield methods, as well a series of preliminary qualitative outcomes, which are also discussed.

The June 2015 version of eRHIC ERL (Fig. 1) is considered in the present report which, from that standpoint, is seen also as a follow on of optical studies regarding an earlier version of eRHIC optics [4], and as such can serve for optics as well as beam and spin dynamics comparisons as eRHIC parameters evolve.

June 2015's version of the ERL is based on a $1.322 \mathrm{GeV}$ linac, and on two FFAG loops located alongside RHIC ('FFAG1' and 'FFAG2' in the sequel). FFAG1 recirculates the electron bunches 4 times on the way up and 4 times on the way down $(0.012 \stackrel{\text { up }}{\rightarrow} 5.300 \stackrel{\text { down }}{\rightarrow} 0.012$, step $1.322 \mathrm{GeV})$, FFAG2 recirculates the electron bunches 12 times up and 11 times down (23 circulating beams, from 6.622 to $21.164 \mathrm{GeV}$ and down back to $6.622 \mathrm{GeV}$. Additional details regarding the FFAG optics based eRHIC concept can be found in Ref. [5].

The electron beam is taken here at linac entrance at $5.3 \mathrm{GeV}$, and the FFAG2 loop only is considered (the reason being the greater effects of synchrotron radiation on beam and spin dynamics), hence the end-to-end cycle discussed :

$$
5.3 \stackrel{\text { up }}{\longrightarrow} 21.164 \stackrel{\text { down }}{\longrightarrow} 5.3 \mathrm{GeV}
$$

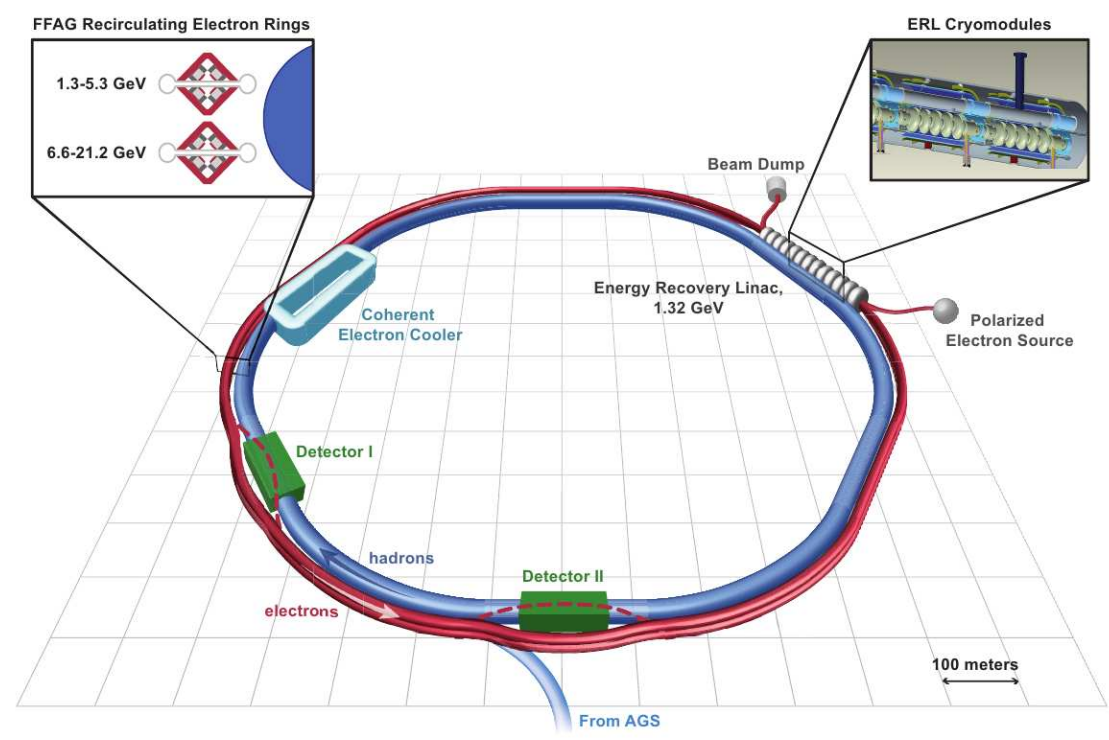

Figure 1: eRHIC ERL with its two recirculation loops alongside RHIC. The top left box shows a cross-section of the FFAG1 (low energy) and FFAG2 (high energy) recirculating loops. The $1.322 \mathrm{GeV}$ linac is located in RHIC IR2, it is connected to the FFAG loops by a merger section (resp. spreader) at its upstream (resp. downstream) end.

In the following, basic properties of the FFAG2 recirculating loop are first investigated (Sec. 2), from a tracking simulation viewpoint essentially. Then some aspects of three additional components of the ERL are discussed, namely the linac and the spreader and merger sections (Sec. 3). Finally all these pieces are assembled and the optics of the ensemble out of the computer model is discussed (Sec. 4). These investigations are concluded with the simulation of a complete $5.3 \stackrel{\text { up }}{\rightarrow} 21.164 \stackrel{\text { down }}{\rightarrow} 5.3 \mathrm{GeV}$ acceleration-deceleration cycle of a 6D bunch (Sec. 5). The input data files for the eRHIC ERL tracking simulations as discussed in this Note, in particular in Sec. 4, are available at

$$
\text { /home/owl/fmeot/zgoubi/struct/bnl/eRHIC/eRHIC_Notes/Note_49 }
$$



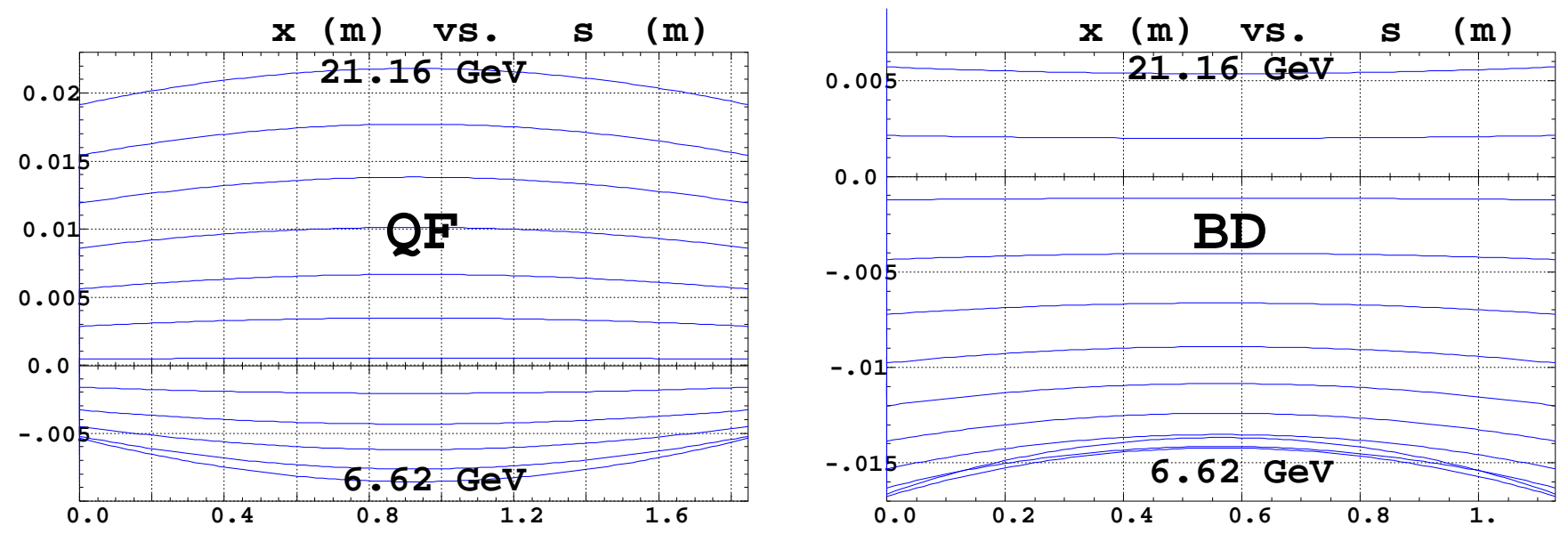

Figure 2: Transverse excursion of the 12 periodic orbits across the FFAG cell magnets, shown in the respective magnet frames ( $\mathrm{x}=0$ is the quadrupole axis). The optical axes of the quadrupoles in the arc cell are radially shifted by $13.48 \mathrm{~mm}$ with respect to one another, this ensures $8.73 \mathrm{mrad}$ orbit bending across the cell.

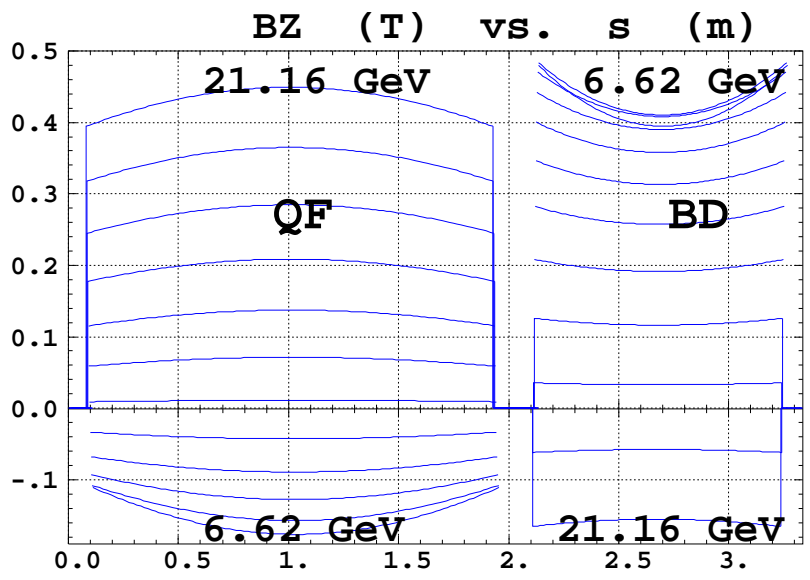

Figure 3: Magnetic field experienced along the 12 orbits, in the hard-edged model.

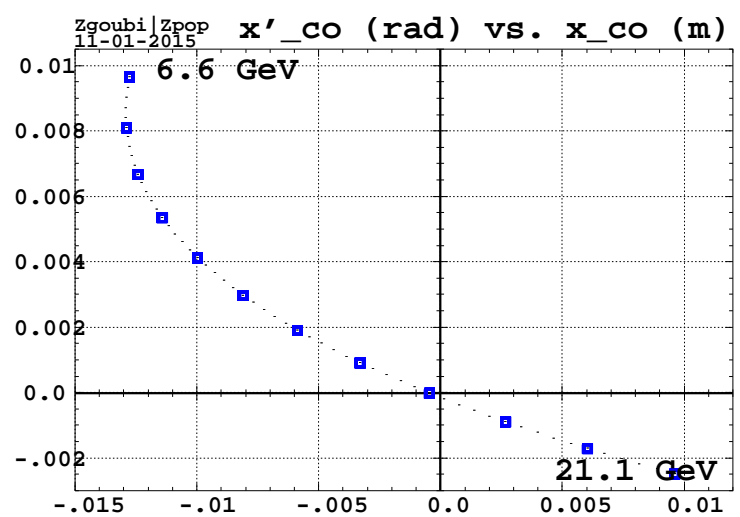

Figure 4: Evolution of the FFAG2 orbits in phase space, as observed at the center of the drift downstream of QF in an arc cell. The blue empty rectangles correspond to the 12 design energies.

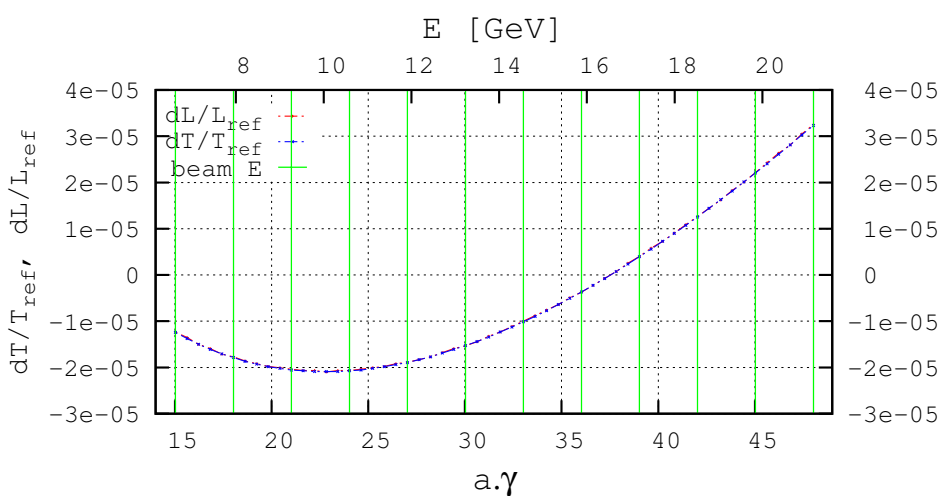

Figure 5: Parabolic variation (relative) of the cell periodic orbits length and time of flight. The reference values are for $16.5 \mathrm{GeV}$, namely, $L_{\text {ref }}=3.36239 \mathrm{~m}, T_{\text {ref }}=11.2157 \mathrm{~ns}$. The vertical bars correspond to the 12 design energies. 


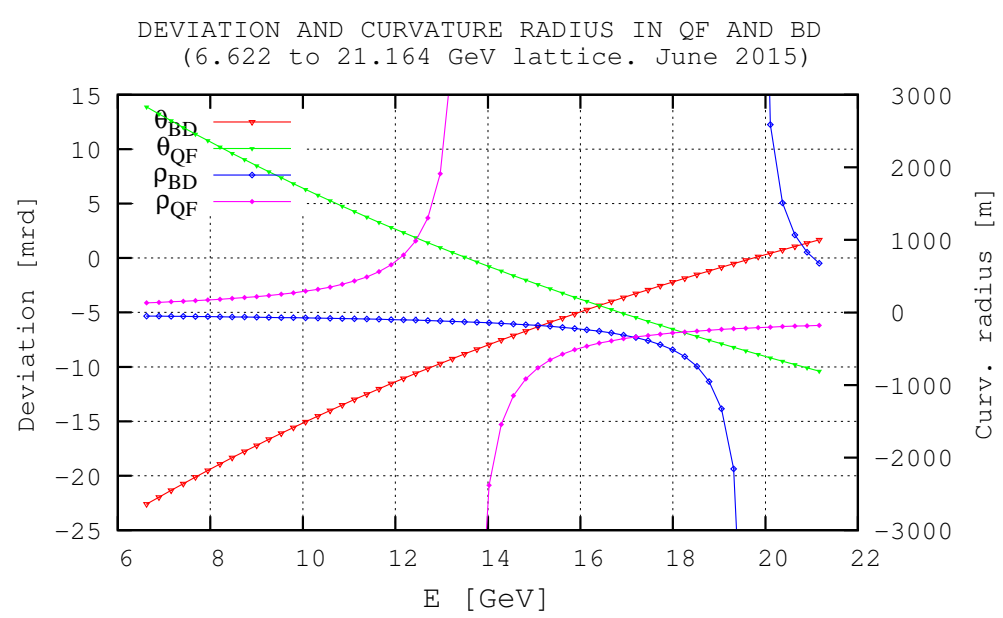

Figure 6: Energy dependence of orbit deviation angle and average curvature radius in arc cell quadrupoles. $\rho_{Q F}$ is large on the $13.2 \mathrm{GeV}$ orbits, close to QF axis, see Fig. 3-left, $\rho_{B D}$ is large on the 19.8 and $18.5 \mathrm{GeV}$ orbits, close to BD axis, Fig. 3-right.

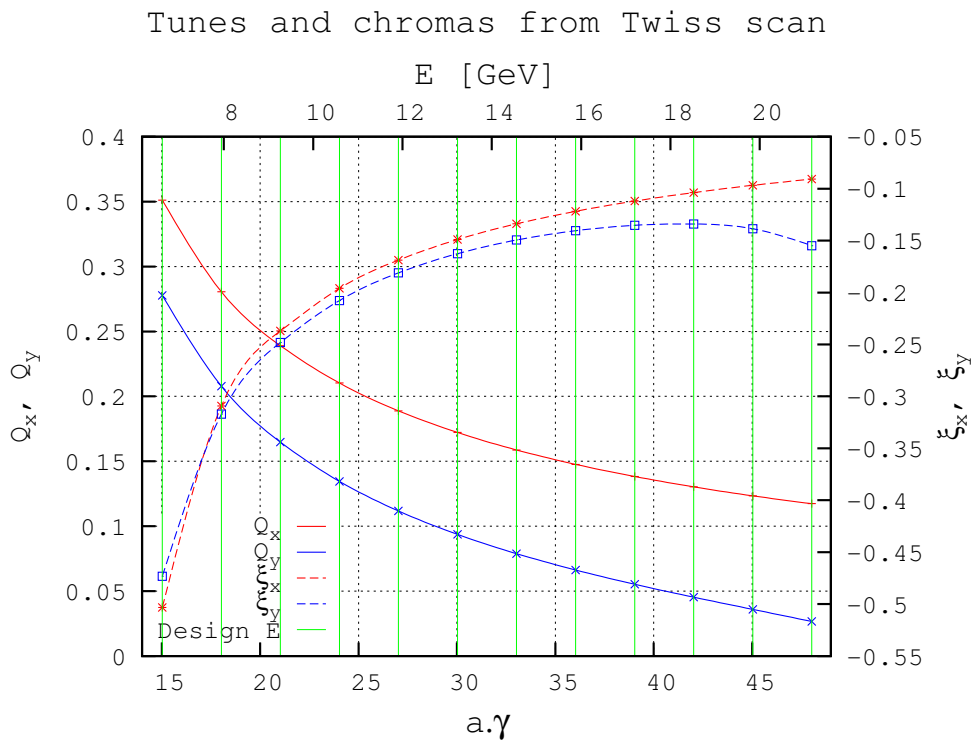

Figure 7: Cell tunes and chromaticities versus energy; the vertical bars materialize the 12 design energies. These are part of the principles of the linear FFAG cell : tunes decreasing with energy (following in that the focusing strength) since the quadrupole gradients are fixed, and natural chromaticity, decreasing with energy.
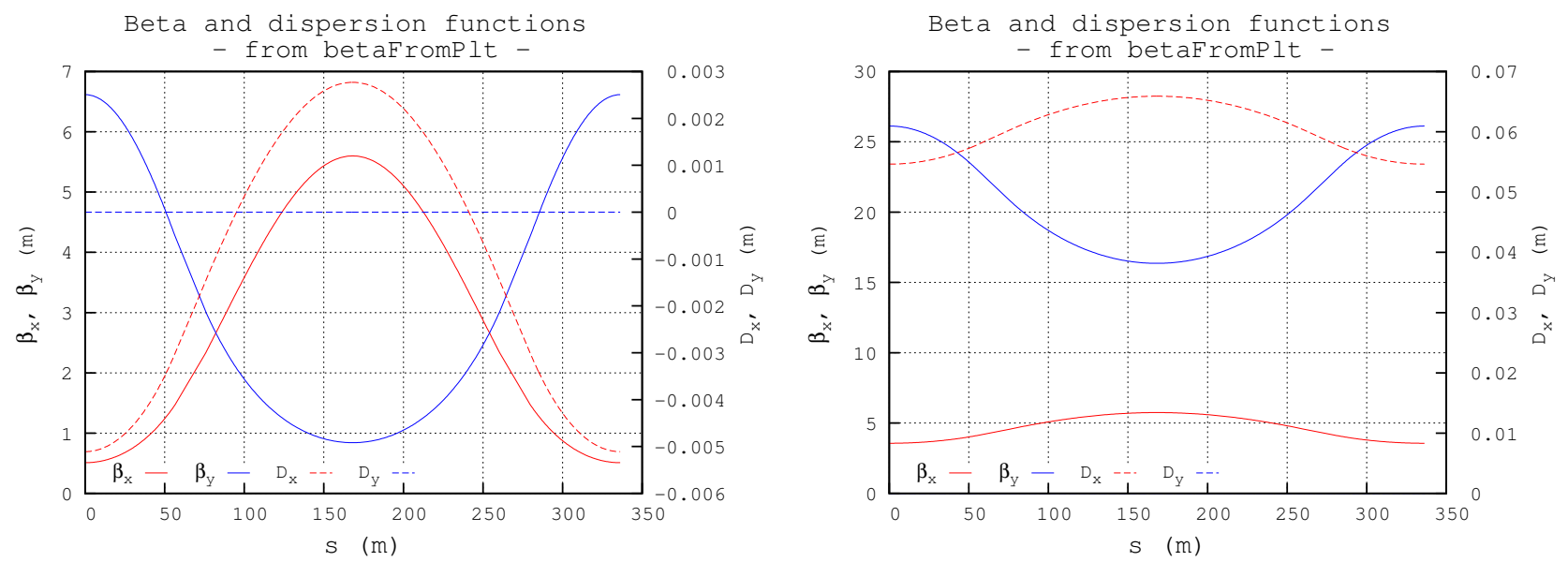

Figure 8: Optical functions at $6.622 \mathrm{GeV}$ (left) and $21.164 \mathrm{GeV}$ (right), from stepwise ray-tracing across the FFAG2 cell. 


\section{FFAG2 recirculation loop}

We first summarize the basic optical properties of the lattice cell (Sec. 2.2). Then the effects of synchrotron radiation (energy loss and spreading, emittance growth) are evaluated turn-by-turn in the FFAG2 loop (Sec. 2.3). (Details of the Monte Carlo method in Zgoubi, and its benchmarking, can be found in [6].) Finally a 23-loop acceleration-deceleration cycle ( 12 beams up from 6.622 to $21.164 \mathrm{GeV}$ and 11 beams back down to $6.622 \mathrm{GeV}$ ) is performed (Sec. 2.4) for additional characterization and also in order to allow comparisons with tracking outcomes in the complete ERL with linac and spreader/merger sections (Sec. 5).

Table 1: Optical parameters of FFGA2 cell. Orbit $\left(x_{c o}\right)$ and beta function values are at the center of BD, cell tunes $\left(Q_{x}, Q_{y}\right)$ and periodic orbit length $(\mathcal{L})$. Due to the symmetry, $x_{c o}^{\prime}=0, \alpha_{x, y}=0$, $D_{x}^{\prime}=0$.

\begin{tabular}{lccccccc}
$\begin{array}{c}\text { Energy } \\
(\mathrm{MeV})\end{array}$ & $\begin{array}{c}x_{c o} \\
\left(10^{-3} \mathrm{~m}\right)\end{array}$ & $Q_{x}$ & $Q_{y}$ & $\begin{array}{c}\beta_{x} \\
(\mathrm{~m})\end{array}$ & $\begin{array}{c}\beta_{y} \\
(\mathrm{~m})\end{array}$ & $\begin{array}{c}D_{x} \\
\left(10^{-2} \mathrm{~m}\right)\end{array}$ & $\begin{array}{c}\mathcal{L} \\
(\mathrm{m})\end{array}$ \\
\hline 6622 & -8.74 & 0.35110 & 0.27778 & 0.5095 & 6.6167 & -5.1090 & 3.362346 \\
7944 & -9.42 & 0.28054 & 0.20789 & 0.8866 & 6.2113 & -2.1110 & 3.362328 \\
9266 & -9.47 & 0.23900 & 0.16481 & 1.2112 & 6.5341 & 1.6730 & 3.362319 \\
10588 & -8.97 & 0.21027 & 0.13456 & 1.5133 & 7.1337 & 6.1010 & 3.362318 \\
11910 & -7.97 & 0.18887 & 0.11175 & 1.8010 & 7.9243 & 1.1057 & 3.362324 \\
13232 & -6.53 & 0.17218 & 0.09370 & 2.0774 & 8.8993 & 1.6450 & 3.362336 \\
14554 & -4.70 & 0.15873 & 0.07887 & 2.3443 & 10.0895 & 2.2205 & 3.362354 \\
15876 & -2.51 & 0.14763 & 0.06628 & 2.6028 & 11.5624 & 2.8261 & 3.362375 \\
17198 & -4.87 & 0.13829 & 0.05528 & 2.8537 & 13.4429 & 3.4570 & 3.362401 \\
18520 & 2.79 & 0.13030 & 0.04533 & 3.0975 & 15.9732 & 4.1090 & 3.362430 \\
19842 & 5.85 & 0.12339 & 0.03598 & 3.3348 & 19.6897 & 4.7787 & 3.362462 \\
21164 & 9.15 & 0.11735 & 0.02660 & 3.5659 & 26.1247 & 5.4633 & 3.362497 \\
\hline
\end{tabular}

\subsection{Structure of the FFAG2 loop}

The structure of the simulated FFAG2 loop is the following :

- It has 6 arcs and 6 long straight sections, following in that RHIC 6-periodicity,

- An arc is comprised of 102 identical BD-drift-QF-drift doublet cells with quadrupole optical axes radially shifted by $13.48 \mathrm{~mm}$ with respect to one another to ensure the bending (see detailed geometry data in App. A, p. 29),

- Five of the six long straight sections (LSS) are identical and each comprised of a string of 52 such cells with quadrupole axes superimposed, these LSS are dispersion free, all energies share a common optical axis, coinciding with quadrupole axes,

- The dispersion suppressors (DS) between the arcs and these five LSS are comprised of 18 of these very cells, with quadrupole axes shifting gradually from zero at the LSS end, to $13.48 \mathrm{~mm}$ at the arc end. Five of these 10 DS sections take the 23 beams (12 recirculations up and 11 down) from their respective FFAG optical axes in the arcs onto their common axis in the downstream LSS, the other five DS have the reverse functionality,

- The remaining LSS (RHIC IR2 region) is occupied by the $120 \mathrm{~m}, 42$ cavity linac and the spreader and merger lines (there are no energy loss neither energy spread compensation cavities in the present simulations),

- Both start and end points of an arc are at the center of a BD magnet in these simulations, for convenience,

- The 12 spreader lines at their downstream end as well as the 12 merger lines at their upstream end are matched to the 12 sets of FFAG orbit optical functions at the center of the arc cell BD magnet (values in Tab. 1), 
- The spreader at its upstream end and the merger at its downstream end are matched to the optical functions at linac ends,

- The IR6 and IR8 transport sections to collision points at top energy $(21.164 \mathrm{GeV})$ are not accounted for,

- Path length adjustments (path length is energy dependent in the FFAG arcs, see Tab 1 and Fig. 5) are taken care of in the spreader and merger sections,

- On top of what precedes, some artifacts are introduced regarding 6D positioning of the bunch at entrance to these various FFAG2 loop sections, this will be addressed in due place.

\subsection{Characteristics of the arc cell}

The geometry of FFAG2 cell is detailed in Fig. 2, additional details can be found in the input data file to Zgoubi in App. A.

The optical properties of the cell are summarized in a series of figures, as follows.

- Figs. 2 and 3 show respectively the transverse excursion of, and magnetic field along, periodic orbits across the arc cell, for the 12 recirculated energies. It can be observed that the field varies in a substantial fashion along the orbit inside a quadrupole at large excursion,

- Fig. 4 : orbit coordinates in phase space, as observed at the center of the $\mathrm{QF} \rightarrow \mathrm{BD}$ drift ( $s=2.04 \mathrm{~m}$ location in Fig. 3),

- Fig. 5 : parabolic energy dependence of orbit length and time of flight,

- Fig. 6 : energy dependence of the deviation angle and average curvature radius in the two quadrupoles. These quantities are obtained from the actual orbit deviation as produced by the stepwise ray-tracing across the BD and QF cell magnets,

- Fig. 7 : tunes and chromaticities,

- Fig. 8 : optical functions across the cell at lower and higher energies.

In these figures $a \gamma$ is the spin precession rate, with $a=1.16 \times 10^{-3}$ the electron anomalous gyromagnetic factor.

\subsection{Synchrotron radiation effects, turn-by-turn}

\subsubsection{Working hypotheses}

Turn-by-turn tracking is performed here, without linac neither any spreader and merger sections. The FFAG2 recirculating loop in this first approach has the following 6-periodic form :

$$
\text { FFAG2 }=6 \times[\text { DS }- \text { LSS }- \text { DS }- \text { ARC }]
$$

The essential differences compared to the ERL structure tracked in Sec. 5 are that,

(i) the linac section has been replaced here by an LSS,

(ii) there are no spreader/merger sections, the structure is 6-periodic and closes onto itself.

Besides, perfect optical alignments are assumed all around the FFAG2 loop.

\subsubsection{Orbit outcomes}

Figure 9 shows the twelve orbits along FFAG2 as defined in Eq. 1 (actually, a plot of the centroid position $\overline{x\left(s_{f}\right)}$ of a 5000-particle bunch), for $\mathrm{E}=6.622 \mathrm{GeV}$ to $\mathrm{E}=21.164 \mathrm{GeV}$, step $1.322 \mathrm{GeV}$. The 12 orbits are all aligned on $\mathrm{x}=0$ along the 6 long straight sections, whereas in the 6 arcs they feature an energy dependent excursion as discussed earlier (Figs. 2, 4). 


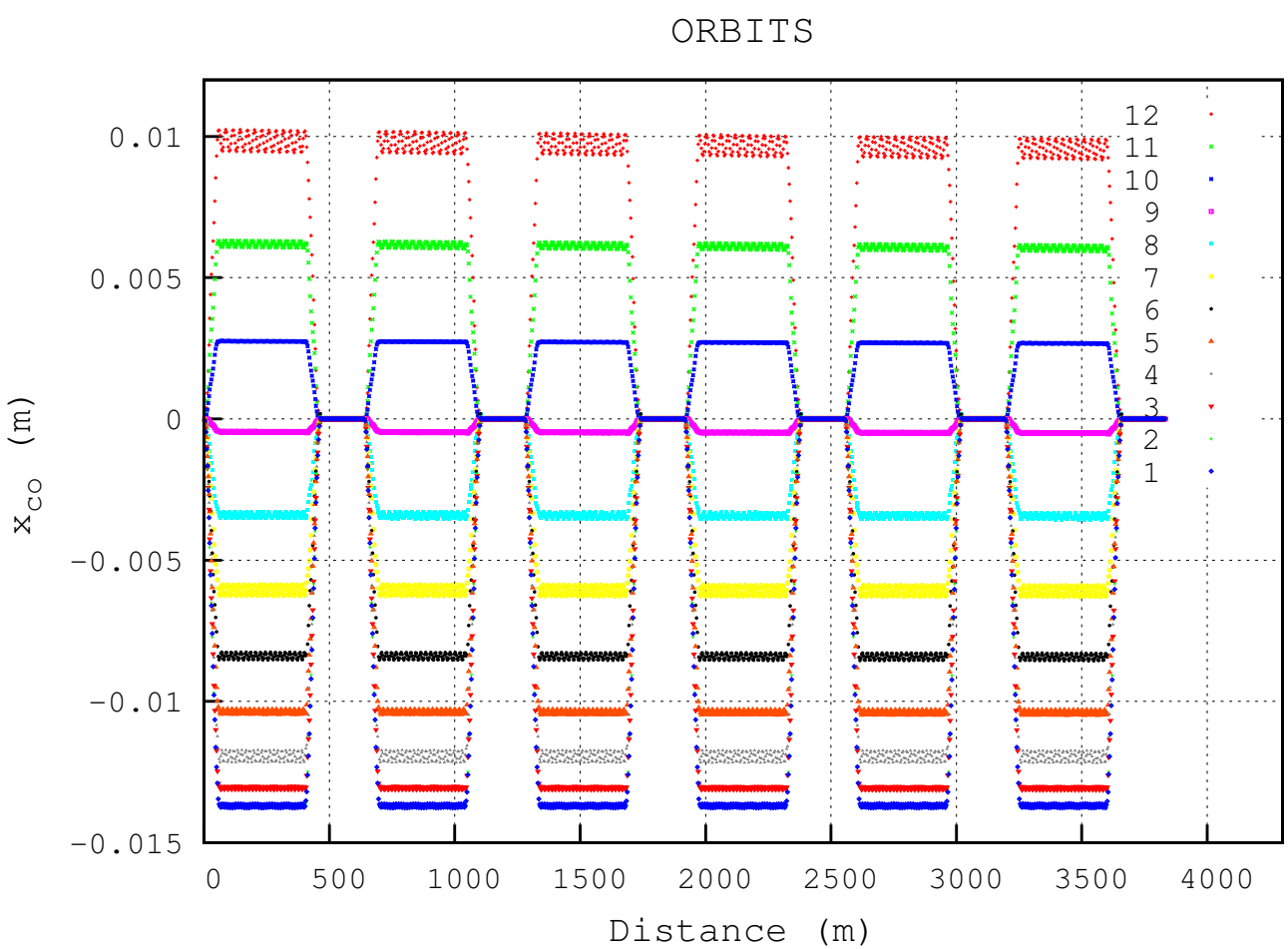

Figure 9: Orbits along the FFAG2 recirculation loop, for the 12 energies concerned : from bottom to top, $\mathrm{E}=6.622$ to $21.164 \mathrm{GeV}$, step $1.322 \mathrm{GeV}$. Along the 6 long straight sections all energies have a common optical axis $(\mathrm{x}=0)$. In the arcs the orbit excursion is recorded in the $\mathrm{QF} \rightarrow \mathrm{BD}$ drift, the $\lesssim \mathrm{mm}$ excursion observed here at all energies (greater at higher energy) is due to a residual orbit mismatch at the dispersion suppressors.

ENERGY LOSS OVER A TURN $(21.16 \mathrm{GeV})$

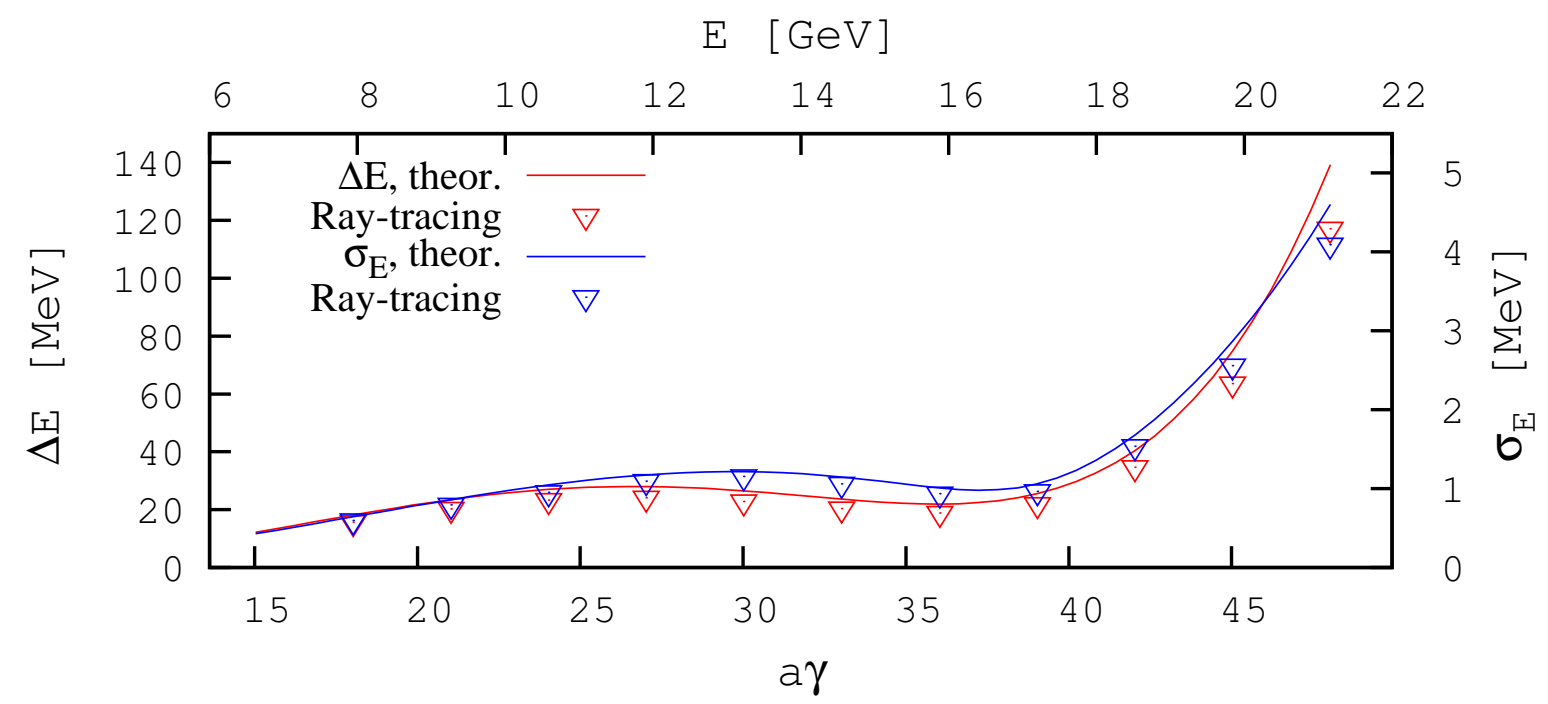

Figure 10: Energy dependence of the single-turn average energy loss (left axis) and energy spread (right axis) in FFAG2 recirculation loop at the 12 recirculated energies (empty triangles). Solid lines ("theor.") are for a $6 \times 120$ cells ring, they are obtained from Eq. 3. The lower SR loss in the FFAG2 loop results from the lesser number of cells per arc, 102, not fully compensated by the SR loss in the DS sections (18 cells) at arc ends. 


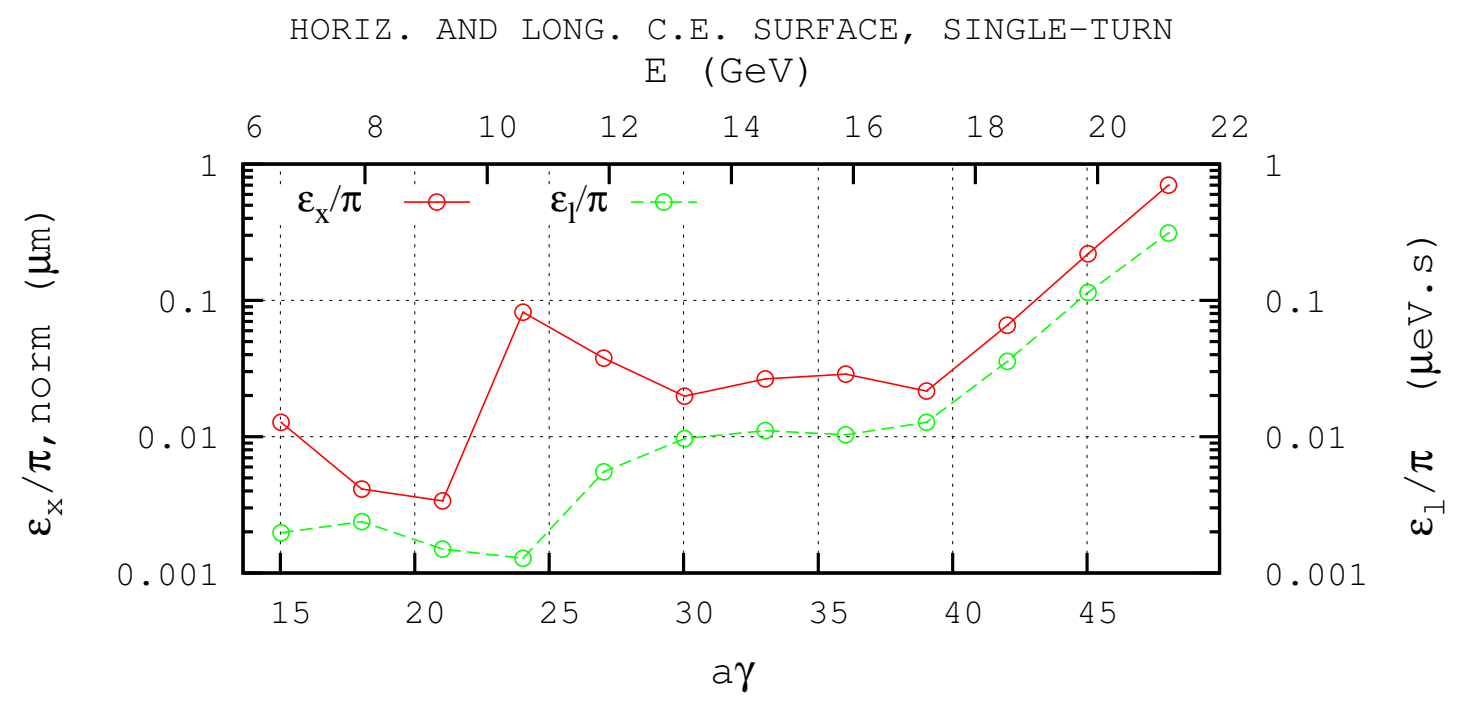

Figure 11: The markers give the single-turn SR induced concentration ellipse surface (the lines are to guide the eye), horizontal normalized (left vertical axis) and longitudinal (right axis). The starting 6D emittance is zero for each energy (a point object). Sample phase space plots are given in Figs. 12, 13. The observation point is at the end of an $\operatorname{LSS}\left(D_{x}\right.$ and $D_{x}^{\prime} \approx 0$ there).

TRANSVERSE

\section{6 (left) and $10.6 \mathrm{GeV}$ (right)}

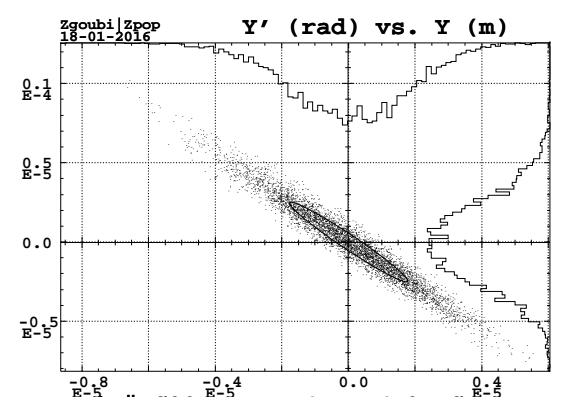

15.9 and $21.2 \mathrm{GeV}$
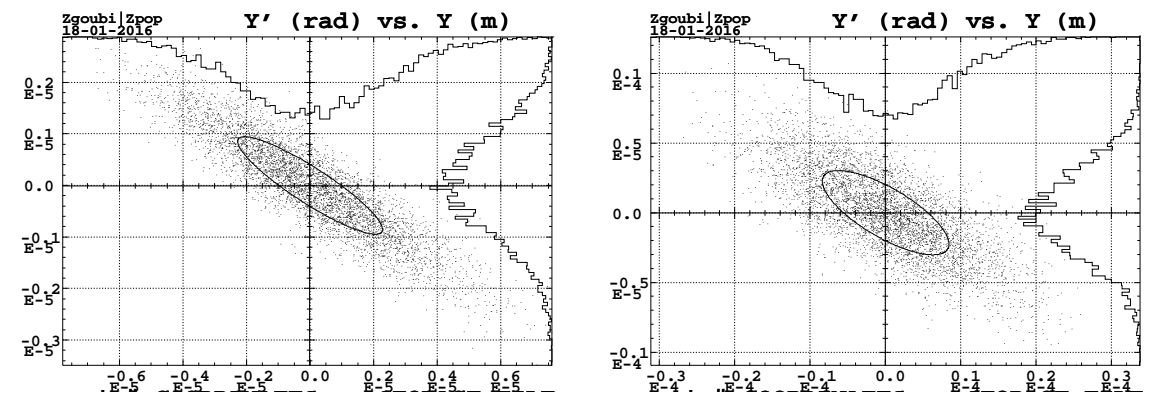

Figure 12: Sample horizontal phase spaces after a FFAG2 loop, as resulting from SR, for 4 different energies. Observation point is at the end of a long straight section. The initial 6D emittance in each case was zero. Chromaticity induced distortion at $10.6 \mathrm{GeV}$ is conspicuous. SR causes an horizontal emittance $\epsilon_{x} / \pi=13 \mathrm{~nm}$ normalized (1 pm geometric) at $6.62 \mathrm{GeV}$, and $\epsilon_{x} / \pi=0.7 \mu \mathrm{m}$ normalized (17 pm geometric) at $21.164 \mathrm{GeV}$.

\section{LONGITUDINAL}

\subsection{GeV}

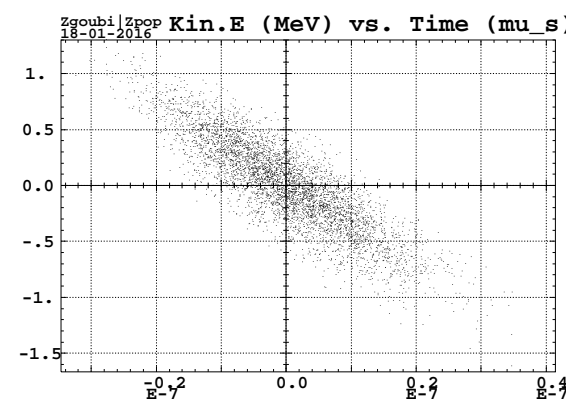

$21.2 \mathrm{GeV}$

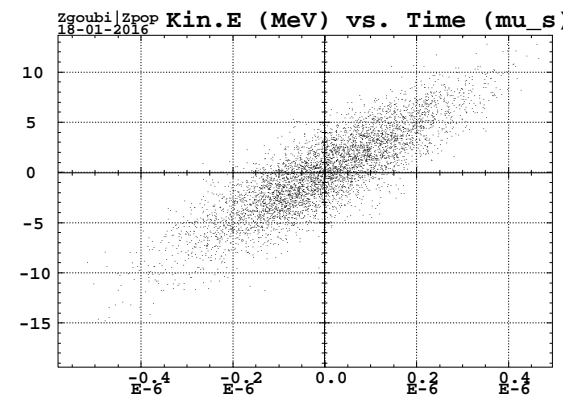

Figure 13: Sample longitudinal phase spaces after a FFAG2 loop, for initial point object. SR causes emittances $\epsilon_{x} / \pi=1 \mu \mathrm{m}$ normalized and $\epsilon_{l} / \pi=1.910^{-9} \mathrm{eV} . \mathrm{s}$ at $6.62 \mathrm{GeV}$, $\epsilon_{x} / \pi=1 \mu \mathrm{m}$ normalized and $\epsilon_{l} / \pi=$ $3.110^{-7}$ eV.s at $21.16 \mathrm{GeV}$. 
A drift of the bunch centroid is conspicuous at highest energy in Fig. 9 (this inward spiraling disappears in the absence of synchrotron radiation). Over a path $s_{i} \rightarrow s_{f}$ it amounts to [7] (to be investigated further),

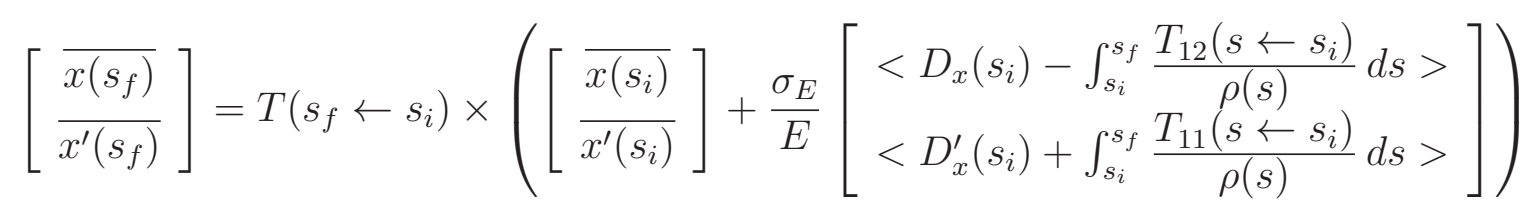

with $\mathrm{D}, \mathrm{D}$ ' the dispersion and its derivative, $\langle *\rangle$ an average over the particle ensemble, $T_{i j}$ first order transport coefficients, and $\sigma_{E}$ the rms energy spread. An orbit oscillation $\lesssim \pm 1 \mathrm{~mm}$ is visible in the arcs (also present in the complete ERL simulation, see Fig. 28, p. 21), this is due to a slight orbit mis-match between LSS and arc across the DS sections, this can be reduced however, the main point being to what degree it has to be to avoid chromaticity induced emittance increase [4].

\subsubsection{SR loss}

Fig. 10 shows the SR induced energy loss and spreading over a FFAG2 loop. These quantities are obtained by tracking a 5000-particle bunch with initial null 6D emittance. The theoretical ("theor.") average energy loss and energy spread in that figure are obtained using [4]

$$
\begin{aligned}
\overline{\Delta E}[\mathrm{MeV}] & =0.96 \times 10^{-15} \gamma^{4}\left(\frac{l_{\mathrm{BD}}}{\rho_{\mathrm{BD}}^{2}}+\frac{l_{\mathrm{QF}}}{\rho_{\mathrm{QF}}^{2}}\right) \times 6 \operatorname{arcs} \times 120 \text { cells } / \operatorname{arc} \\
\sigma_{E} & \approx 1.94 \times 10^{-14} \gamma^{7 / 2} \sqrt{\frac{l_{\mathrm{BD}}}{\left|\rho_{\mathrm{BD}}^{3}\right|}+\frac{l_{\mathrm{QF}}}{\left|\rho_{\mathrm{QF}}^{3}\right|}} \times \sqrt{6 \times 120}
\end{aligned}
$$

with 120 the number of cells (the very cell discussed in Sec. 2.2) necessary to close a circle given the $8.73 \mathrm{mrad}$ single cell deviation (in the FFAG2 loop, the orbit closure is ensured with 102 cells per arc and 18 cells per DS section), and with $l_{\mathrm{QF}}, l_{\mathrm{BD}}$ the magnet lengths and $\rho_{\mathrm{QF}}, \rho_{\mathrm{BD}}$ their average curvature radii as obtained from the stepwise ray-tracing (Fig. 6).

The energy dependence of the SR induced horizontal and longitudinal concentration ellipse surface produced by this 5000-particle tracking is displayed in Fig. 11, corresponding single-turn horizontal and longitudinal phase space portraits are displayed in Figs. 12, 13. Note that the contribution of momentum spread in $\epsilon_{x}$ has not been removed, it is however a small quantity since the dispersion function is negligible at the observation point (i.e., at the end of a long straight section). There is no vertical emittance effect ( $\epsilon_{y}$ remains zero) since the present Monte Carlo SR simulation [6] does not account for the recoil effect (which would however be negligible).

Note that the quantity displayed in Fig. 11 is the surface $\mathcal{S}_{x}(s)=4 \pi \sqrt{\Delta}$ of the concentration ellipse (abusively called "emittance"), defined by

$$
\gamma(s) x^{2}+2 \alpha(s) x x^{\prime}+\beta(s) x^{\prime 2}=\frac{\mathcal{S}_{x}(s)}{\pi}
$$

with $\quad \alpha(s)=-\frac{\overline{x x^{\prime}}(s)}{\sqrt{\Delta}}, \quad \beta(s)=\frac{\overline{x^{2}}(s)}{\sqrt{\Delta}}, \quad \gamma(s)=\frac{\overline{x^{\prime 2}}(s)}{\sqrt{\Delta}}, \quad \Delta=\overline{x^{2}}(s) \overline{x^{\prime 2}}(s)-{\overline{x x^{\prime}}}^{2}(s)$

(wherein $\overline{u v}$ denotes the average $\frac{1}{n} \sum_{i=1}^{n}(u-\bar{u})(v-\bar{v})$ ). As a consequence a deformation of the particle distribution in phase space (such as observed for instance in Fig. 12 at $10.6 \mathrm{GeV}$, a chromatic effect in this case) may induce a change in that quantity whereas the beam emittance would actually not change.

\subsection{3-loop up-down cycle in a simplified, 6-arc ring}

Still in order to get a sense of orders of magnitude, we conclude this preliminary approach to the ERL simulation with an up-down tracking in an even simpler loop, a 6-arc ring, comprised of $6 \times 120$ cells, with, at a single location, a linac simulation by a thin-lens $1.322 \mathrm{GeV}$ boost. 
A particle bunch is, in a row, accelerated in 11 linac passes up (12 recirculation loops), from 6.622 to $21.164 \mathrm{GeV}$ and decelerated in 11 linac passes (11 recirculation loops) down back to $6.622 \mathrm{GeV}$. The following artifacts are included in the simulation :

after each turn, prior to tackling the next one,

(i) SR loss is compensated at the linac by giving a turn-dependent energy kick $1.322+\Delta E$ with $\Delta E$ computed from Eq. 3,

(ii) the bunch is re-centered in position and angle on the theoretical FFAG orbit once per turn, next to the boost (following the energy dependence of the orbit as displayed in Fig 4).

Two simulations are performed :

(1) A first one with starting 6D emittance zero (point object), the evolution of the horizontal and of the longitudinal emittances are displayed in Fig. 14. The vertical emittance remains zero since photon recoil is not accounted for in the Monte Carlo SR simulation. Some values can be found in Tab. 2 regarding the cumulated effect of SR otherwise summarized in Fig. 14.

Fig. 15 (resp. Fig. 16) displays the horizontal (resp. longitudinal) phase space at $21.16 \mathrm{GeV}$ (after 12 recirculations up) and back down to $6.62 \mathrm{GeV}$ (after an additional 11 linac passes, decelerating).

Regarding the evolution of longitudinal emittance, in addition to energy spread as given by Eq. 3 which has been shown to behave as expected, bunch lengthening over the $\left[s, s_{f}\right]$ distance, resulting from the stochastic energy loss, is expected to satisfy [7],

$$
\sigma_{l}=\left(\frac{\sigma_{E}}{E}\right)\left[\frac{1}{L_{\text {bend }}} \int_{s}^{s_{f}}\left(D_{x}(s) T_{51}\left(s_{f} \leftarrow s\right)+D_{x}^{\prime}(s) T_{52}\left(s_{f} \leftarrow s\right)-T_{56}\right)^{2} d s\right]^{1 / 2}
$$

with the integral being taken over the bends, $D_{x}$ and $D_{x}^{\prime}$ the dispersion function and its derivative, $T_{5 i}$ the first order trajectory lengthening coefficients (indices 5 and $i=1,2,6$ stand for respectively $\delta l, x, x^{\prime}, \delta p / p$ ). This is to be investigated further.

(2) A second one with starting bunch emittance $\sim 50 \mu \mathrm{m}$ normalized transverse, both planes, momentum spread random uniform in $\pm 3 \times 10^{-4}$, bunch length zero, the evolution of the horizontal and of the longitudinal emittances are displayed in Figs. 17, 18 respectively. In that simulation different numbers of particles have been tried to test the convergence ( $1 \mathrm{k}, 5 \mathrm{k}$ and $10 \mathrm{k})$, as well as two different integration step sizes in the two quadrupoles $(1 \mathrm{~cm}$ and $3 \mathrm{~cm})$. The relative effect is small, the difference is essentially in a slight translation of the curves. Some values can be found in Tab. 2 regarding the cumulated effect of SR otherwise summarized in Figs. 17, 18.

Table 2: Some values (orders of magnitude) regarding the cumulated effect of SR on transverse and longitudinal motions, at top energy and back to $6.62 \mathrm{GeV}$, in a $6 \times 120$ cell ring. The "6.622 (start)" rows give the initial conditions in the two tracking simulations.

\begin{tabular}{lccccc}
\hline $\begin{array}{l}\text { bunch energy } \\
(\mathrm{GeV})\end{array}$ & $\begin{array}{c}\epsilon_{x} / \pi, \text { norm. } \\
(\mu \mathrm{m})\end{array}$ & $\begin{array}{c}\epsilon_{y} / \pi, \text { norm } \\
(\mu \mathrm{m})\end{array}$ & $\begin{array}{c}\epsilon_{l} / \pi \\
(\mu \mathrm{eV} . \mathrm{s})\end{array}$ & $\begin{array}{c}\frac{\sigma_{E}}{E} \\
\left(10^{-4}\right)\end{array}$ & $\begin{array}{c}\sigma_{l} \\
(\mathrm{~mm})\end{array}$ \\
\hline $\begin{array}{l}\text { Initial point object } \\
6.622 \text { (start) }\end{array}$ & 0 & 0 & 0 & 0 & 0 \\
21.16 & 2.7 & 0 & 4.2 & 3 & 0.3 \\
6.62 (down end) & 4.4 & 0 & 7.5 & 11 & 0.8 \\
\hline & & & & & \\
Initial extended object & 50 & 50 & 0 & \pm 3, unif. & 0 \\
6.622 (start) & 59 & 50 & 6 & 3 & 0.4 \\
21.16 & 65 & 52 & 10 & 11 & 0.9 \\
6.62 (down end) & & & & & \\
\hline
\end{tabular}




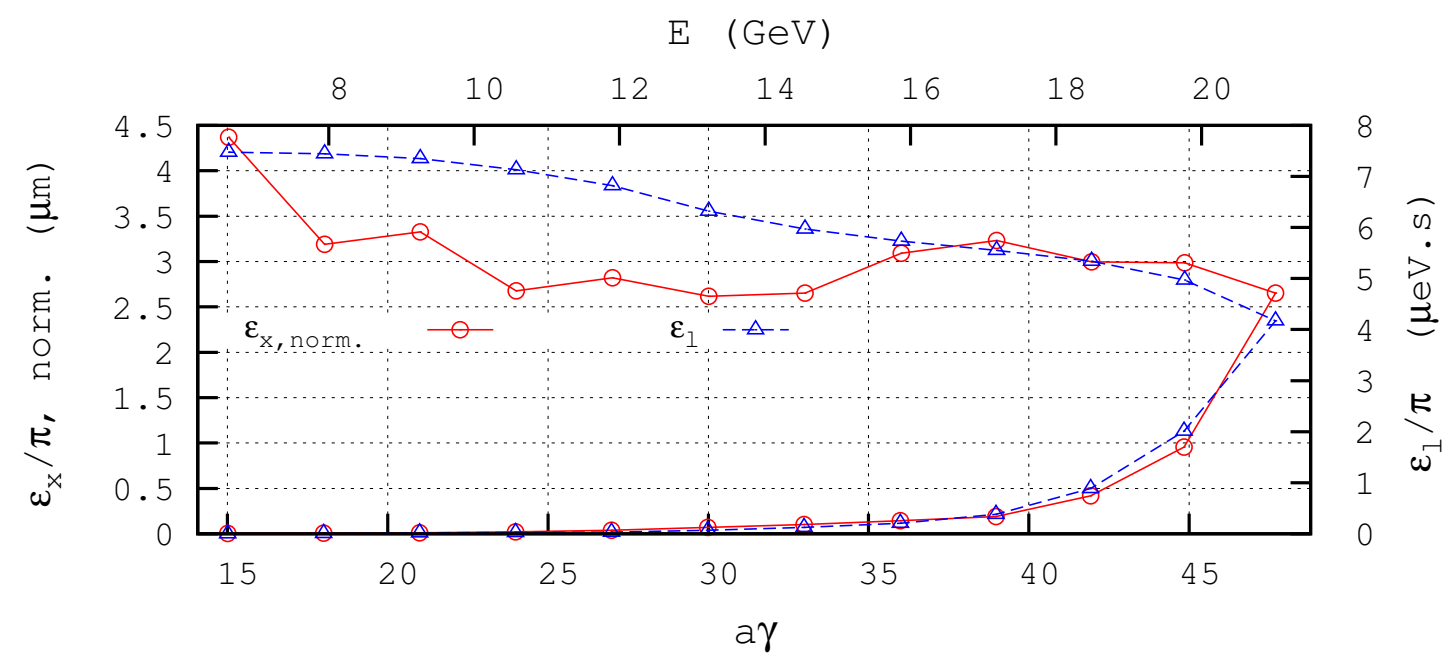

Figure 14: Markers in this figure (lines are to guide the eye) give the evolution of horizontal (left vertical axis) and longitudinal (right axis) bunch emittances under the effect of SR in the case of an initial point object (6D emittance zero), over a 23-loop end-to-end up-down cycle in a simplified 6-arc ring $(6.622 \stackrel{\text { up }}{\rightarrow} 21.164 \stackrel{\text { down }}{\rightarrow} 6.622 \mathrm{GeV})$.

\section{HORIZONTAL}

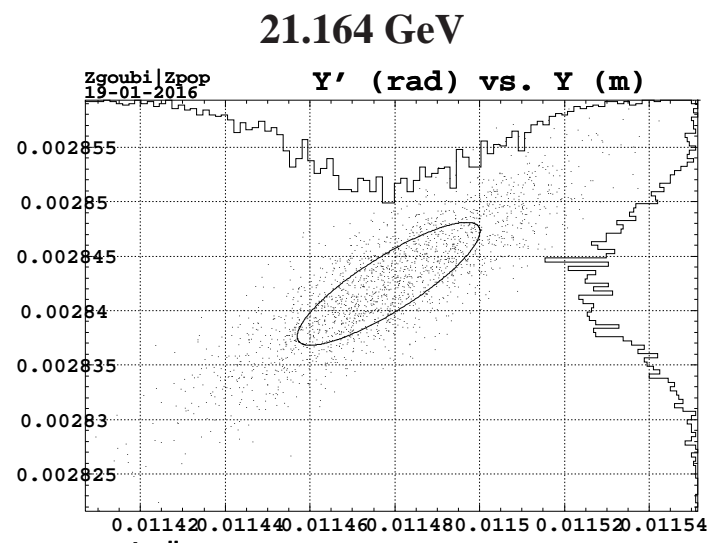

\section{$6.622 \mathrm{GeV}$ DOWN}

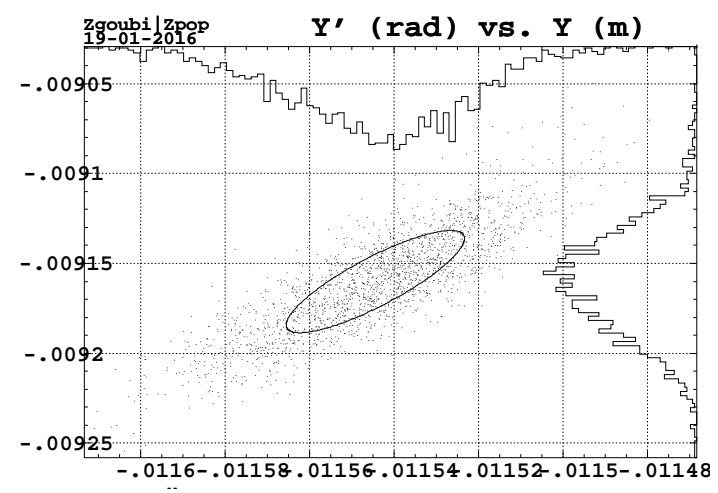

Figure 15: Sample horizontal phase spaces. SR causes an horizontal emittance $\epsilon_{x} / \pi=2.7 \mu \mathrm{m}$ normalized at $21.16 \mathrm{GeV}$, and $\epsilon_{x} / \pi=4.4 \mu \mathrm{m}$ back to $6.62 \mathrm{GeV}$. In both cases, horizontal (left col.) and longitudinal (right col.), phase space portraits are at $21.16 \mathrm{GeV}$ after 12 loops (top), and back down to $6.62 \mathrm{GeV}$ after deceleration (bottom). Observation point is at the thin-lens linac. The initial $6 \mathrm{D}$ emittance in each case was zero.

\section{LONGITUDINAL}

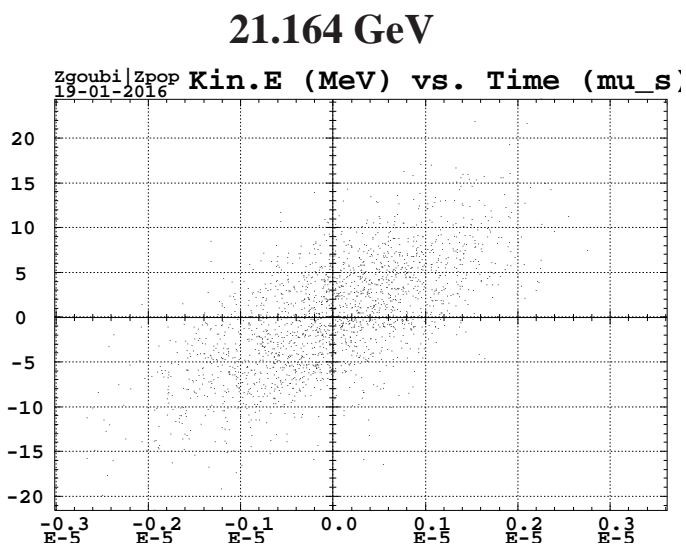

6.622 GeV DOWN

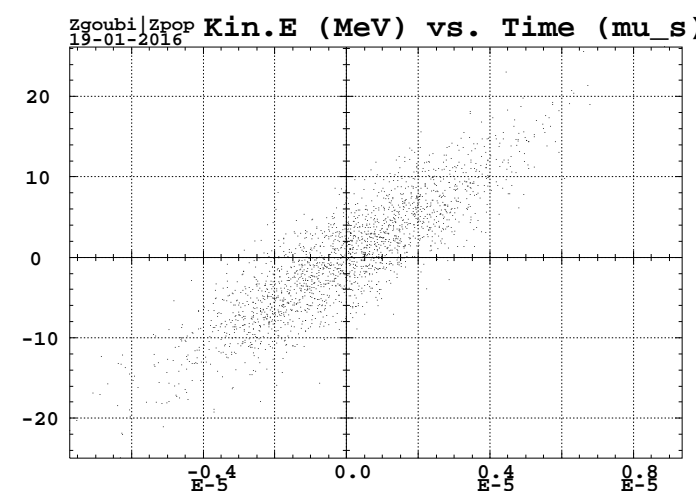

Figure 16: Sample longitudinal phase spaces. SR causes emittances $\epsilon_{l} / \pi=4.210^{-6} \mathrm{eV} . \mathrm{s}$ at $21.16 \mathrm{GeV}$, and $\epsilon_{l} / \pi=$ $7.510^{-6} \mathrm{eV}$.s back to $6.62 \mathrm{GeV}$. 
TRANSVERSE EMITTANCES

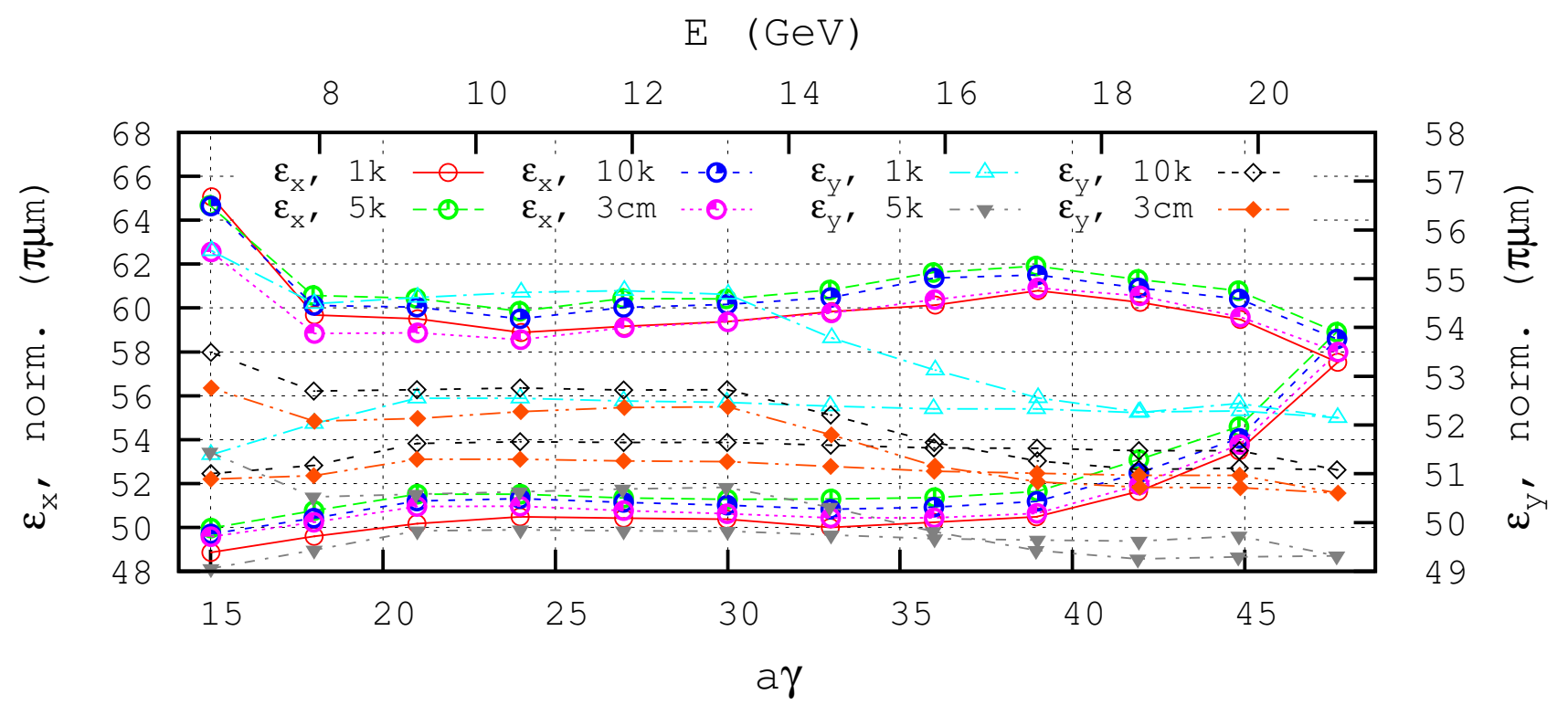

Figure 17: Markers in this figure (lines are to guide the eye) give the evolution of horizontal (left vertical axis) and vertical (right axis) bunch emittances under the effect of SR in the case of initial conditions $\epsilon_{x}=\epsilon_{y}=50 \pi \mu \mathrm{m}$ normalized, $\delta E / E$ uniform random in $\pm 310^{-4}$ and $\sigma_{l}=0$. The bunch is tracked over a 23-loop end-to-end up-down cycle in a simplified 6-arc ring $(6.622 \stackrel{u p}{\rightarrow} 21.164 \stackrel{\text { down }}{\rightarrow} 6.622 \mathrm{GeV})$. The various curves correspond to either a different number of tracked particles $\left(1,5\right.$ or $\left.10 \times 10^{3}\right)$, or to different integration step sizes in cell quadrupoles $(1 \mathrm{or} 3 \mathrm{~cm})$.

\section{LONGITUDINAL EMITTANCE}

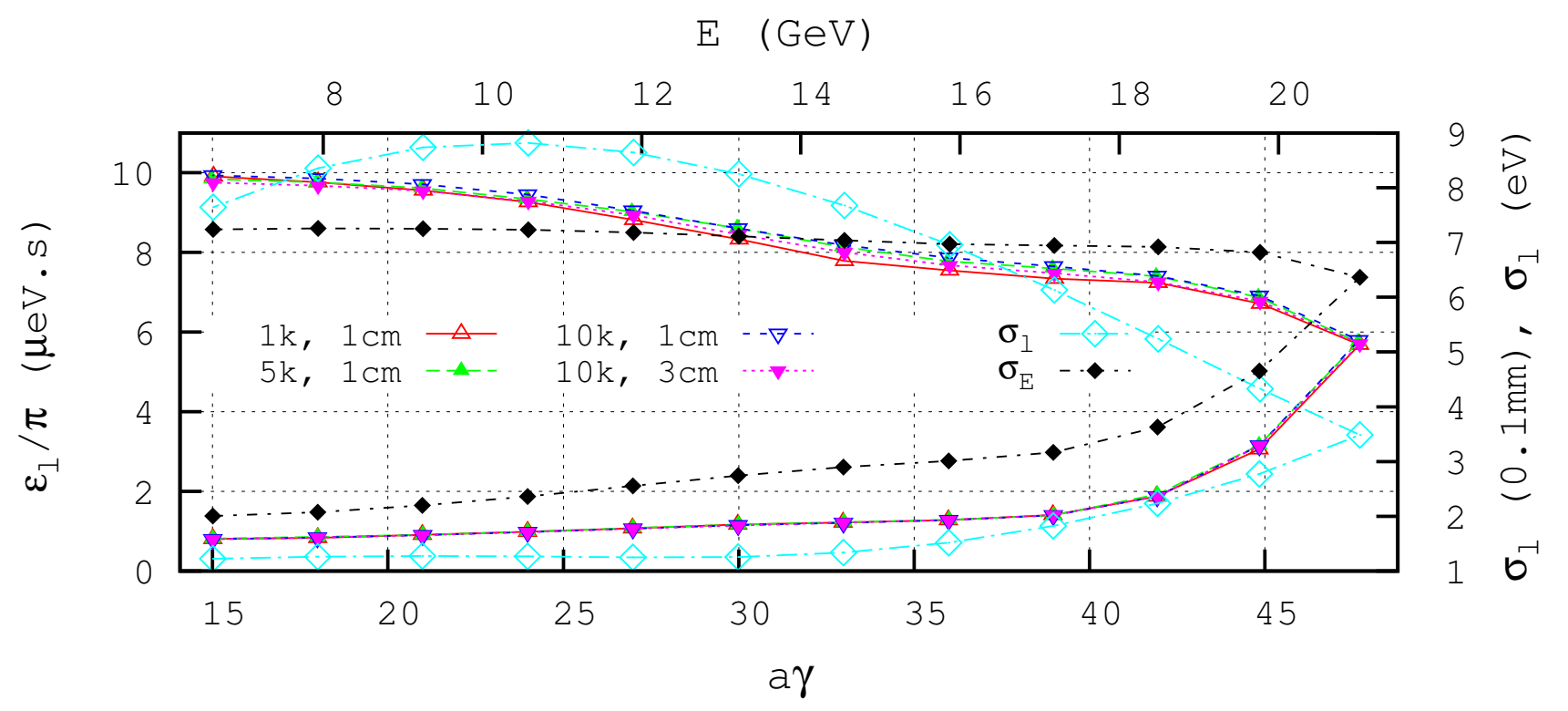

Figure 18: Evolution of longitudinal bunch emittance (left axis, left four markers) and $\sigma_{l}, \sigma_{E}$ (right axis, right two markers), for initial conditions $\epsilon_{x}=\epsilon_{y}=50 \pi \mu \mathrm{m}$ normalized, $\delta E / E$ uniform random in $\pm 310^{-4}$ and $\sigma_{l}=0$. The bunch is tracked over a 23-loop end-to-end up-down cycle in a simplified 6-arc ring $(6.622 \stackrel{\text { up }}{\rightarrow} 21.164 \stackrel{\text { down }}{\rightarrow}$ $6.622 \mathrm{GeV})$. 


\subsection{Polarization}

The spin vector is injected horizontal in the ERL, and precesses around the vertical magnetic field at a rate of $a \gamma \alpha$ ( $\alpha$ is the azimuthal angle) in the course of a recirculation by the FFAG2 loop. The 1.322 GeV linac energy ensures polarization parallel to the longitudinal axis at IP6 and IP8. Due to energy spread, spin precession undergoes spreading ("spin diffusion").

In the following we first assess the effect of SR induced energy spread on spin diffusion (Sec. 2.5.1). Then we assess spin diffusion for a bunch with nominal transverse emittances and momentum spread (Sec. 2.5.2).

A theoretical approach can be used to check tracking outcomes, as follows. The solution of the diffusion equations in constant magnetic field writes [8]

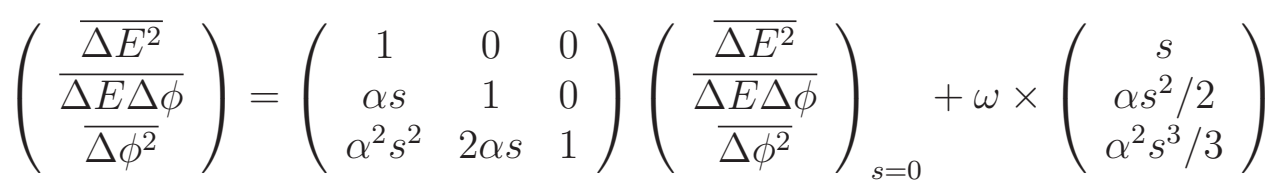

where $s$ is the distance in the field, $\omega=\frac{C}{\rho^{3}} \lambda_{c} r_{e} \gamma^{5} E^{2} \approx 1.44 \times 10^{-27} \frac{\gamma^{5}}{\rho^{3}} E^{2}, \alpha=\frac{a}{\rho E_{0}} \approx \frac{1}{0.4406 \rho}$ (with $\lambda_{c}=\hbar / m_{e} c$ the electron Compton wavelength, $C=110 \sqrt{3} / 144, E_{0}=m_{e} c^{2} / e$ the electron rest mass).

Assuming a starting state $\left(\frac{\overline{\Delta E^{2}}}{\overline{\Delta E \phi}}\right)_{s=0}=0$ (this is the case for each energy for instance, in the turn-byturn tracking, Sec. 2.5.1) yields $\sigma_{E}={\frac{s}{\Delta E^{2}}}^{1 / 2}=\sqrt{\omega s}$ (which in passing identifies with the familiar $\sigma_{E} / E=$ $\left.3.810^{-14} \frac{\gamma^{5 / 2}}{\rho^{3 / 2}} \sqrt{s}\right)$, so that

$$
\sigma_{\phi}={\overline{\Delta \phi^{2}}}^{1 / 2}=\sqrt{\frac{\omega \alpha^{2} s^{3}}{3}}=\frac{\alpha s}{\sqrt{3}} \sigma_{E}, \quad \text { or, given } \quad s=2 \pi \rho, \quad \frac{\sigma_{\phi}}{\sigma_{E}}=8.23[\mathrm{rad} / \mathrm{GeV} / \text { turn }]
$$

\subsubsection{Synchrotron radiation effects, turn-by-turn}

Spin tracking is performed here in the conditions of Sec. 2.3, namely, turn-by-turn tracking in the 6-periodic

$$
\text { FFAG2 }=6 \times[\mathrm{DS}-\mathrm{LSS}-\mathrm{DS}-\mathrm{ARC}]
$$

without linac neither any spreader and merger sections.

Tracking results are displayed in Fig. 19. The " $\sigma_{E}$ " curve is that of Fig. 10, for comparison with the spin diffusion angle rms value, $\sigma_{\phi}$. Their ratio takes a quasi-constant value $\sigma_{\phi} / \sigma_{E} \approx 10 \mathrm{rad} / \mathrm{GeV}$ close to the expected $8.23 \mathrm{rad} / \mathrm{GeV}$ (Eq. 5). Note : $a \gamma \alpha$ in that plot appears to differ from an integer multiple of 360 deg (its expected value) by $1 \sim 2 \mathrm{deg}$, this stems from the lack of accuracy of SR energy loss compensation at the linac boost, a small effect, of little relevance here.

\subsubsection{3-loop up-down cycle in a 6-arc ring}

We conclude with spin tracking in the conditions of Sec. 2.4, namely, a simple 6-arc ring is considered, comprised of $6 \times 120$ cells, with, at a single location, a linac simulation by a thin-lens $1.322 \mathrm{GeV}$ boost. A 5000-particle bunch is taken from 6.622 to $21.164 \mathrm{GeV}$ in 11 linac passes. Results are displayed in Fig. 20. The cumulated effect amounts to $\sigma_{\phi} \approx 15$ degrees at the end of the final $21.1 \mathrm{GeV}$ loop (top-left plot). The top-right and bottom plots show that the dominant cause in the matter of final polarization is the injected bunch energy spread. 


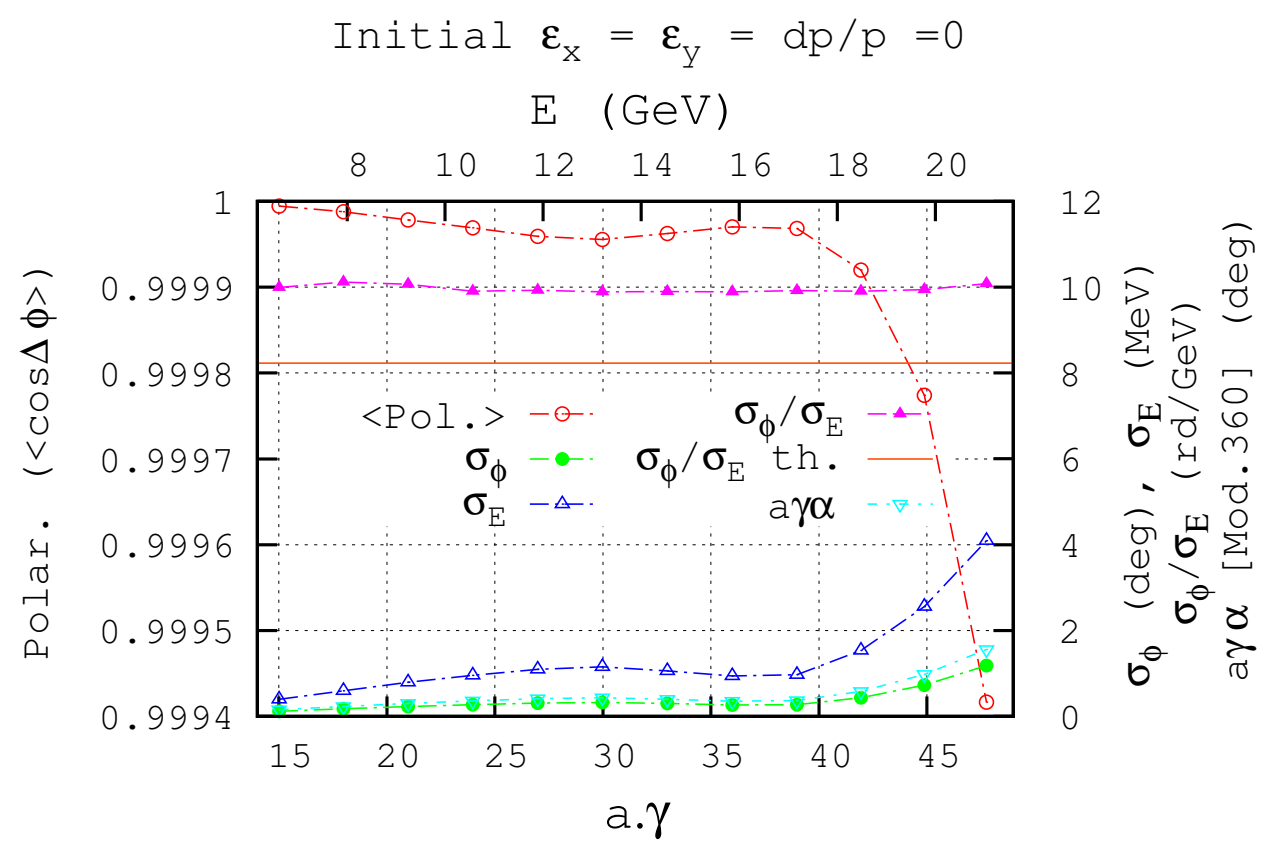

Figure 19: Turn-by-turn in FFAG2 loop (markers; the lines are to guide the eye) : final polarization ( $<\cos \Delta \phi>$, left axis) and spin diffusion $\left(\sigma_{\phi}\right.$, right axis) in a 5000-particle bunch (zero size at start of a turn), for the 12 different energies 6.622 to $21.164 \mathrm{GeV}$, step $1.322 \mathrm{GeV}$.
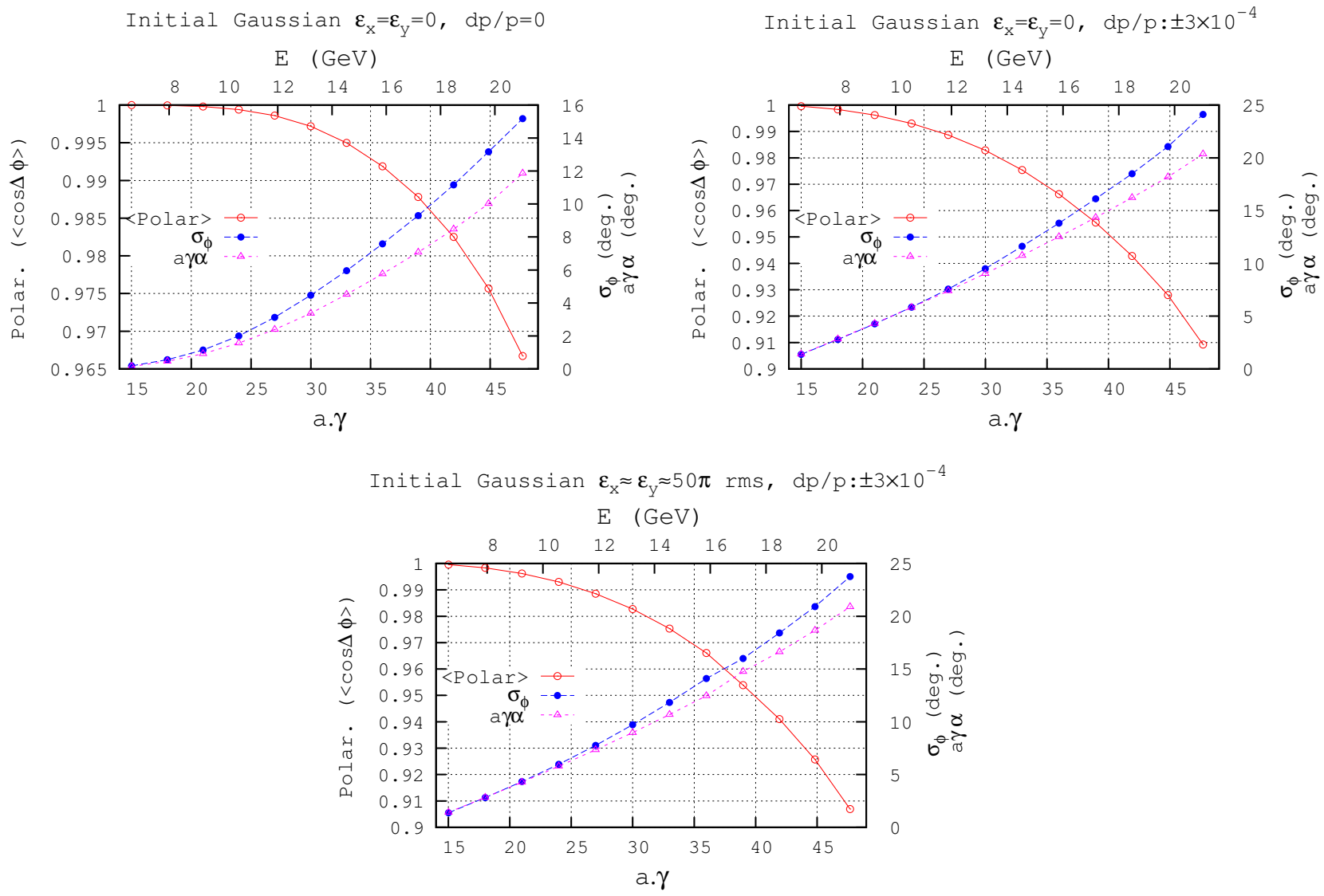

Figure 20: Evolution of cumulated spin diffusion in the case of a 11 linac-pass acceleration cycle (12 complete 6-arc loops), $6.622 \rightarrow 21.164 \mathrm{GeV}$, in a simplified 6-arc ring. 


\section{Three more pieces}

This Section discusses the handling of the three additional pieces needed to complete the ERL, namely, the linac, spreader, and merger sections.

\subsection{Linac}

\subsubsection{Chambers matrices}

Transport through the linac cavities uses "Chambers matrices" [9], the corresponding source code has been copied from the Saclay code BETA [10], where it had been implemented for the design of the "ALS2" linac [11], the interest being the resulting reliability.

These matrices take the following form :

- For both planes (x stands indifferently for $\mathrm{x}$ or $\mathrm{y}$ ) :

$$
\left(\begin{array}{c}
x \\
x^{\prime}
\end{array}\right)_{\text {out }}=\left(\begin{array}{cc}
\cos (u)-\sqrt{2} \sin (u) \cos (\phi) & v W_{i} \sin (u) \cos (\phi) \\
-\frac{\sin (u)}{v W_{o}}\left(2 \cos (\phi)+\frac{1}{\cos (\phi)}\right) & \frac{1}{W_{o} W_{i}}(\cos (u)+\sqrt{2} \sin (u) \cos (\phi))
\end{array}\right)\left(\begin{array}{c}
x \\
x^{\prime}
\end{array}\right)_{i n}
$$

with $u=\log \left(W_{o} / W_{i}\right) /(\sqrt{8} \cos (\phi)), v=\sqrt{8} L_{c a v} /\left(W_{o}-W_{i}\right), W_{i}, W_{o}$ respectively the incoming and outgoing kinetic energies, $L_{\text {cav }}$ the cavity length, $\phi$ the particle phase at the cavity.

- If $\left(W_{o}-W_{i}\right) / W_{i} \ll 1$ the matrix is used under the simplified form

$$
\left(\begin{array}{c}
x \\
x^{\prime}
\end{array}\right)_{\text {out }}=\left(\begin{array}{cc}
\left.\sqrt{(} W_{i} / W_{o}\right) & \left.L_{\text {cav }} \times \sqrt{(} W_{i} / W_{o}\right) \\
0 . & \left.\sqrt{(} W_{i} / W_{o}\right)
\end{array}\right)\left(\begin{array}{c}
x \\
x^{\prime}
\end{array}\right)_{\text {in }}
$$

- The code allows working with determinant 1 matrices, obtained by renormalizing the transport coefficients by the square root of the matrix determinant.

\subsubsection{Tracking particles on invariants}

This is a preliminary test. It uses the structure and beam conditions in the ERL simulations, next Sections, namely :

- a 42 cavity linac, $\mathrm{L}=120 \mathrm{~m}$,

- cavity parameters $L_{\text {cav }}=1.7749 \mathrm{~m}$, voltage $314.762 \mathrm{MV}$, RF frequency $422.26 \mathrm{MHz}$,

- launch point is at linac entrance,

- symmetric beta functions are considered (this is an arbitrary choice), namely, $\alpha_{i / o}=1, \beta_{i / o}=L=120 \mathrm{~m}$, resulting in minimum $\beta=60 \mathrm{~m}$ at linac center, $\mathrm{s}=\mathrm{L} / 2$.

Typical tracking results consistent with spreader and merger optical settings in the ERL (this is discussed in Sec. 3.2) are displayed in Fig. 21.

\subsubsection{Bunch transport}

We complete this preliminary "benchmarking" of the dynamics along the linac with Figs. 21, 23, which display (satisfactory) sample results for a 2000-particle bunch transport, with starting paraxial transverse conditions and $\delta E / E$ uniform random in $\pm 10^{-4}, \sigma_{l}=2 \mathrm{~mm}$. Note that the transverse and longitudinal phase spaces so obtained also constitute a reference for comparison with transport outcomes, for a nominal size bunch, through the complete ERL as discussed further in Sec. 5.1. 

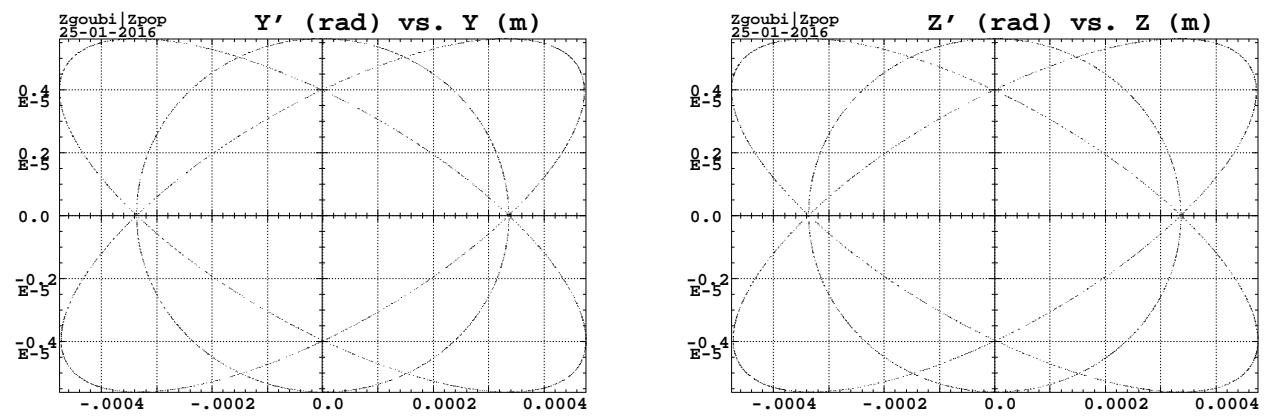

Figure 21: An initial 1000-particle bunch with all transverse particle coordinates taken on a fixed invariant $\gamma x^{2}+2 \alpha x x^{\prime}+\beta x^{\prime 2}=\epsilon / \pi$ (x stands for $\mathrm{x}$ or $\mathrm{y} ; \epsilon_{x}=\epsilon_{y}=25 \pi \mu \mathrm{m}$, normalized, $6.622 \mathrm{GeV}$ here) is tracked from entrance to exit of the linac. The figure shows horizontal (left plot) and vertical (right plot) phase spaces at entrance (converging ellipse), middle (circle) and exit (diverging ellipse).
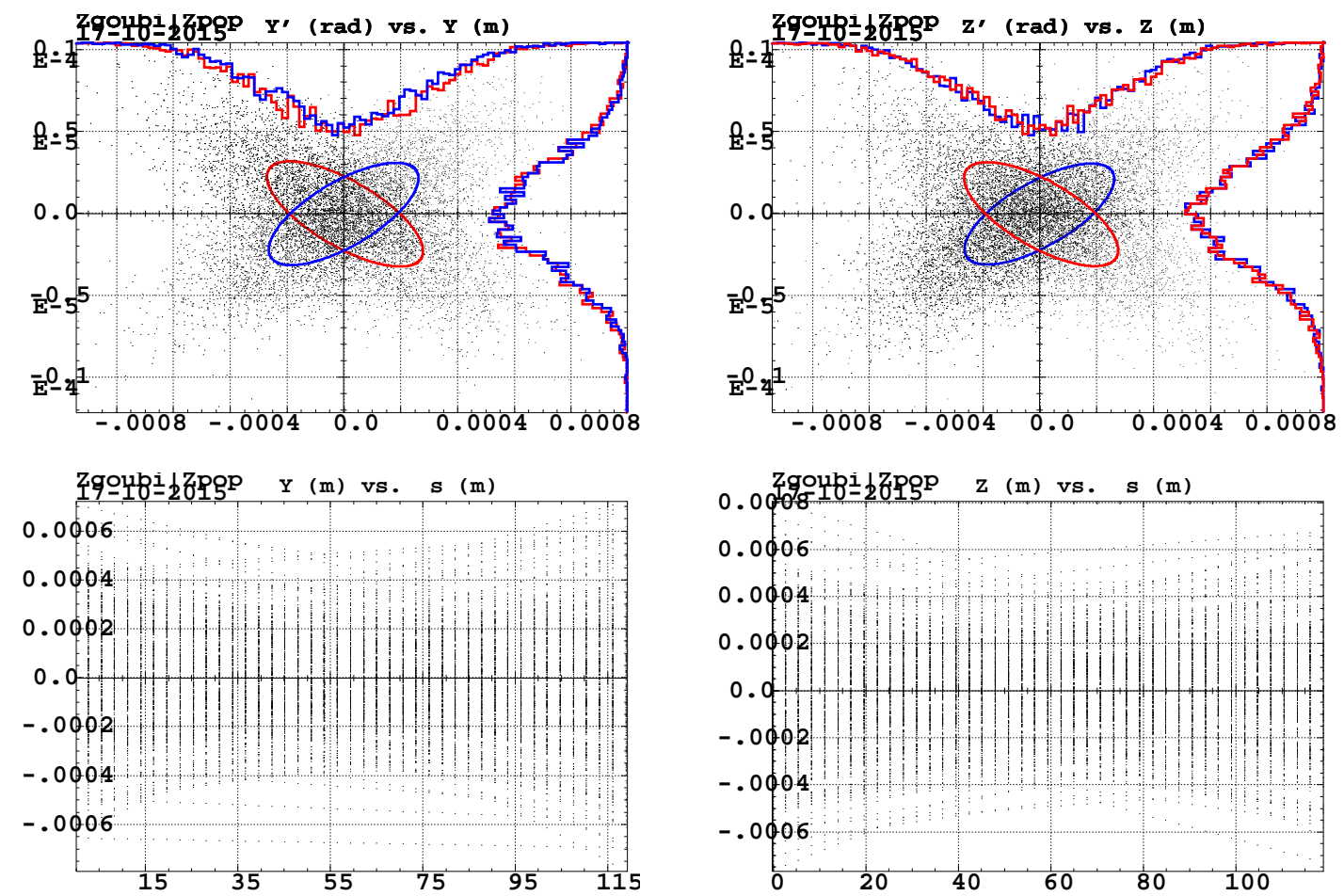

Figure 22: Top : horizontal (left) and vertical (right) transverse phase space at linac ends (red $r m s$ ellipse : linac entrance, $19.8 \mathrm{GeV}$; blue rms ellipse : exit, $21.2 \mathrm{GeV}$ ). Bottom: horizontal (left) and vertical (right) beam bundle through the linac feature equal beam amplitude at both ends, and minimized waist at center.
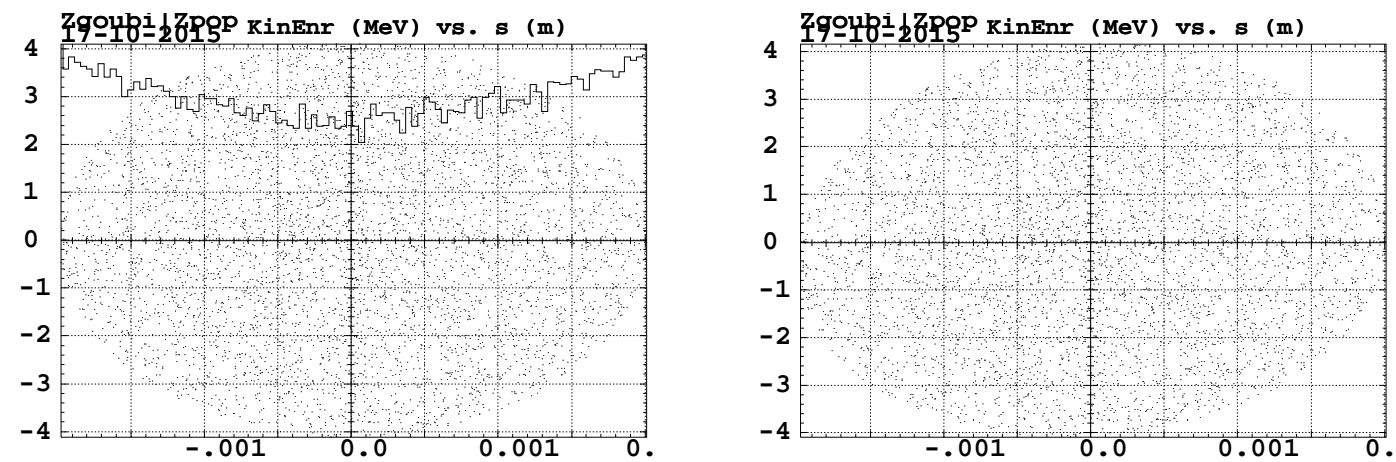

Figure 23: Longitudinal phase space in (left, 19.8 GeV) and out of linac (right, 21.2 GeV). 


\subsection{Spreader and merger sections}

The 12 spreader lines (linac to FFAG2 arc) and 12 merger lines (FFAG2 arc to linac) in the ERL ensure a series of optical functions : orbit positioning, optical matching between linac and FFAG2 loop (i.e., beta functions and horizontal dispersion which is non-zero on FFAG side), path length (as it is energy dependent in the FFAG loop) and R56 adjustments.

In these simulations, see next Secs. 4, 5, we use a single design for all spreader and merger lines, namely, the 21.164 design shown in Fig. 24, symmetrized for spreader or merger use. Using that very line at different rigidities is essentially, model wise, a matter of scaling fields with energy. One consequence though is that, except for the $21.164 \mathrm{GeV}$ spreader and merger lines, SR effects as well as spin dynamics can't be evaluated (bending radii, possible presence of a vertical chicane, and some other aspects, have to be optimized separately (e.g., SR has to be minimized) for each spreader/merger line). Note that the design of the spreader/merger lines is done in such a way as to minimize chromaticity induced emittance growth (possibly by adding sextupoles), this is under study.

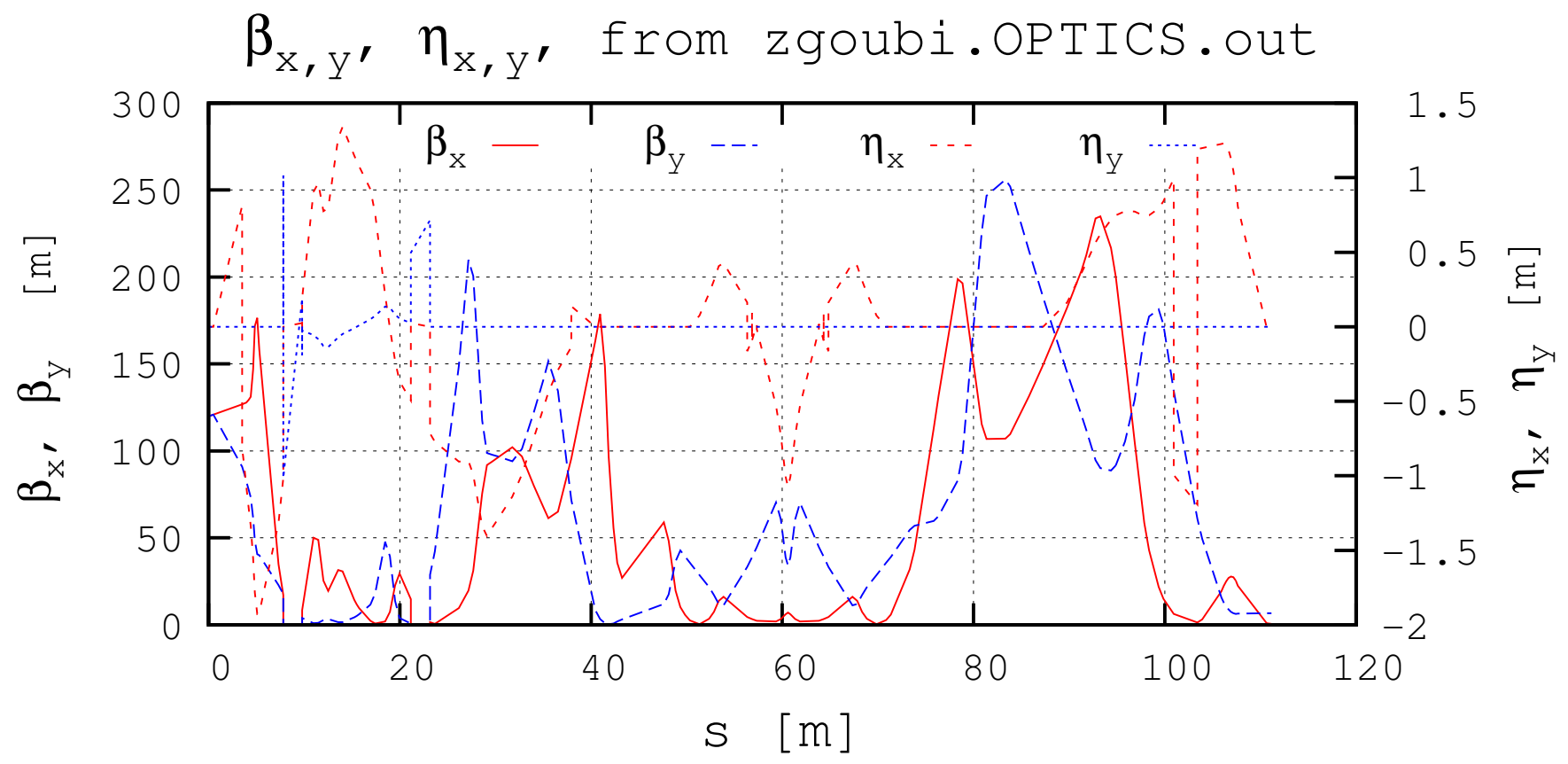

Figure 24: Optical functions in the $21.164 \mathrm{GeV}$ spreader line (connected to the linac to the left, to the FFAG2 loop to the right). $\beta_{x}=\beta_{y}=120 \mathrm{~m}$ and $\alpha_{x}=\alpha_{y}=1$ to the left, and (see Tab. 1) $\alpha_{x}=\alpha_{y}=0, \beta_{x}=3.5659 \mathrm{~m}, \beta_{y}=26.1247 \mathrm{~m}$, $D_{x}=5.46 \times 10^{-2} \mathrm{~m}, D_{x}^{\prime}=0$ to the right. The symmetric of this line is taken for the $21.164 \mathrm{GeV}$ merger. That very line is used for all energies, with magnet settings scaled accordingly. Note : discontinuities are observed in the optical functions, these are artifacts, located at, and due to, $\pi / 2$ rotations (vertical bends) and $\pi$ rotations (negative horizontal bends).

\section{ERL optics, complete}

The lattice in the up-down ERL tracking simulations has the following form

ERL

$$
=\underbrace{\begin{array}{c}
\text { Observation point } \\
\text { merger }+\downarrow \text { linac }+ \text { spreader }
\end{array}}_{\text {RHIC IR2 region }}+\text { FFAG2 }
$$

with 


$$
\mathrm{FFAG} 2=\mathrm{ARC}-\mathrm{DS}-\frac{1}{2} \mathrm{LSS}+\underbrace{\left[\frac{1}{2} \mathrm{LSS}-\mathrm{DS}-\mathrm{ARC}-\mathrm{DS}-\frac{1}{2} \mathrm{LSS}\right]}_{\mathbf{4} \text { times }}+\frac{1}{2} \mathrm{LSS}-\mathrm{DS}-\mathrm{ARC}
$$

and

$$
\mathrm{ARC}=102 \times\left[\frac{1}{2} \mathrm{BD}-\mathrm{drift}-\mathrm{QF}-\mathrm{drift}-\frac{1}{2} \mathrm{BD}\right]
$$

Note in particular, compared to the "simplified 6-arc" simulations in Secs. 2.3 and 2.5.1, the absence of DS sections in IR2 region in this complete ERL layout (actually not fully complete, see below, but close enough that it delivers a qualitative overview of the ERL model to be eventually achieved, and of various outcomes to be expected).

Some more details regarding the optical structure in this simulation of the complete ERL :

- An arc is comprised of 102 identical doublet cells (Eq. 11) with quadrupole optical axes radially shifted by $13.48 \mathrm{~mm}$ with respect to one another to ensure $8.73 \mathrm{mrad}$ bending per cell (optical properties as described in Sec. 2.2, geometry details in App. A, p. 29).

- Five long straight sections (LSS) are comprised of a string of 52 such cells with quadrupole axes superimposed instead. These LSS are dispersion free, all energies share a common optical axis (as in Fig. 9), aligned on quadrupole axes.

- The dispersion suppressors (DS) between arcs and each one of the five LSS are comprised of 18 of these cells, yet with quadrupole axes shifting gradually from zero at their LSS end, to $13.48 \mathrm{~mm}$ at their arc end. Six of these DS take the 23 beams (12 recirculations up, 11 down) from their respective FFAG optical axes in the arcs onto their common axis in the downstream LSS, the other 6 DS have the reverse functionality.

- The remaining straight section is occupied by the $120 \mathrm{~m}, 42$ cavity linac and the spreader and merger lines (along RHIC IR2 region, see Fig. 1). There are no energy loss neither energy spread compensation cavities in the present simulations.

- Both start and end points of an arc are at the center of a BD magnet (Eq. 11), for convenience.

- The spreader at its downstream end as well as the merger at its upstream end

- steer the beam respectively onto and from the (non zero) FFAG orbits (see Figs. 2, 4, p. 4),

- are matched to the optical functions at the center of the arc cell BD magnet (Tab. 1).

- The spreader at its upstream end and the merger at its downstream end are matched to the optical functions and dispersion at linac ends.

- The beam transport to the IPs at IR6 and IR8 at top energy (21.164 GeV) is not accounted for, instead the $21.164 \mathrm{GeV}$ recirculation is treated like a regular one, simply taking the bunches back to deceleration phase for energy recovery.

- Path length adjustments (path length is energy dependent in the FFAG arcs, see Fig. 5) are taken care of in the spreader and merger sections.

Perfect optics alignments are assumed everywhere. Moreover, artificial 6D positioning of the bunch is introduced at various locations, this will be addressed in detail in due place. Note also, in the following simulations the entrance point to the linac is the starting point of the optical sequence in Zgoubi, the "Observation point" in Eq. 9.

The optics properties are summarized in Figs. 25-28, they appear to be well maintained compared to the individual optical module properties as discussed earlier (i.e., cell and FFAG2 loop, Secs. 2.1, 2.2 ; linac, Sec. 3.1 ; spreader/merger, Sec. 3.2). 


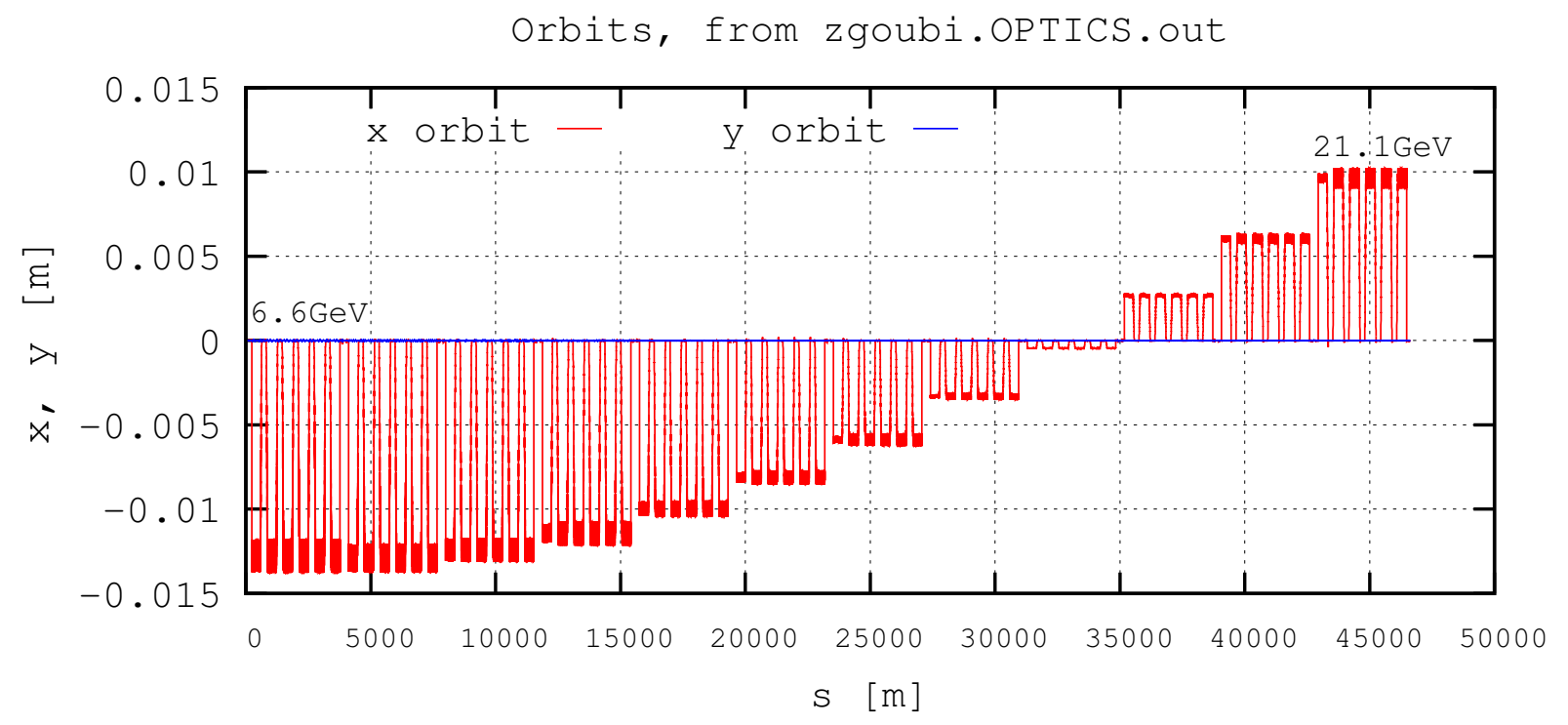

Figure 25: This figure shows the 12 recirculated orbits (obtained by actually tracking a single particle) from 6.622 to $21.164 \mathrm{GeV}$, a $12 \times 3.887 \mathrm{~km}$ long path. Each one of the 12 "steps" in this plot represents a complete ERL turn (Eq. 9), $3.887 \mathrm{~km}$ long. In the arcs the orbit behaves as detailed in Fig. 9, with excursion ranging from $\sim-1.35 \mathrm{~cm}$ at $6.622 \mathrm{GeV}$ (left hand end) to $\sim+0.9 \mathrm{~cm}$ at $21.164 \mathrm{GeV}$ (right hand end). In the 5 long straight sections between the arcs and in the linac straight between two "steps", the orbit coincides with the $\mathrm{x}=0$ axis in the figure.

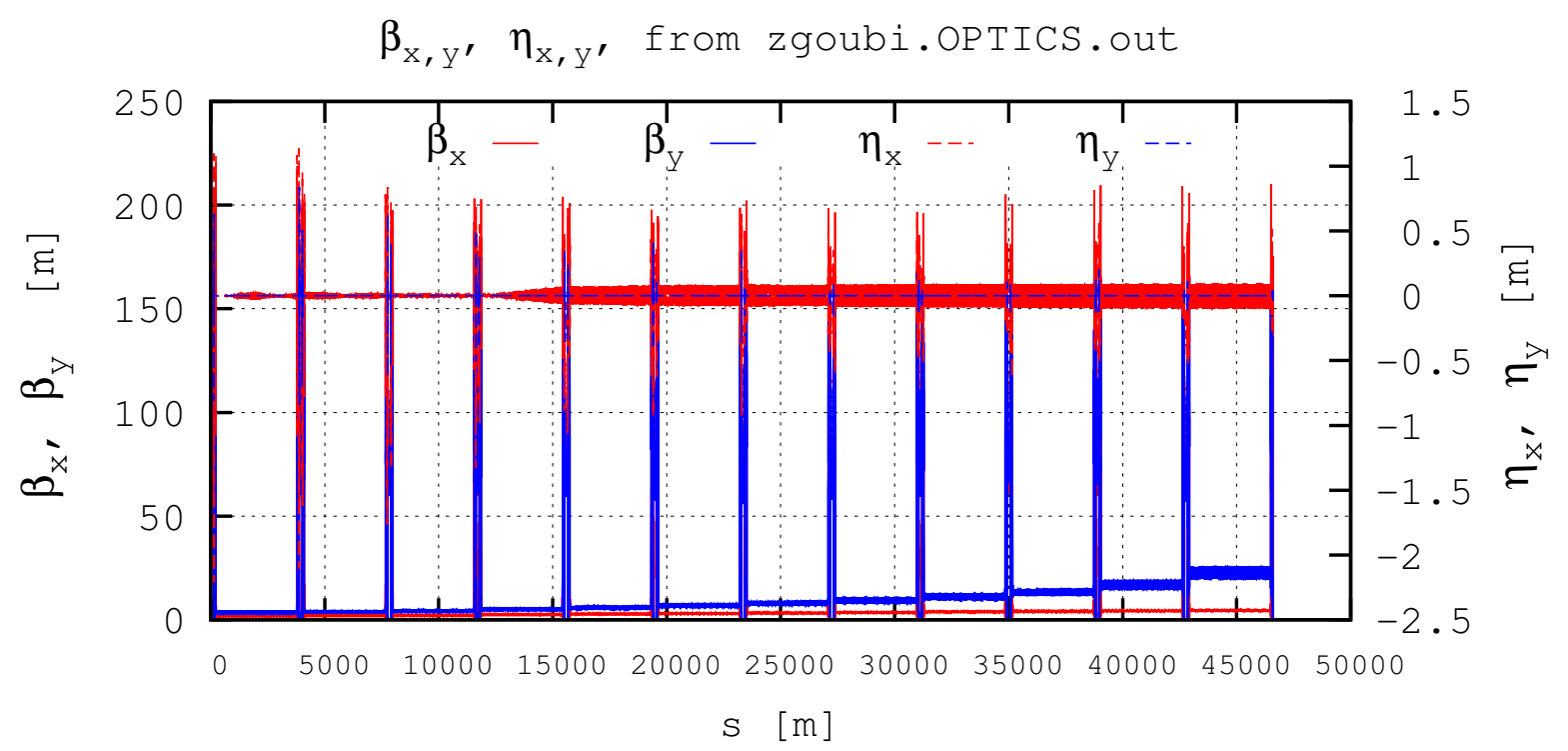

Figure 26: This figure shows the betatron functions (computed from the transport of 11 sample particles) from 5.3 to $21.164 \mathrm{GeV}$. The $\sim 200 \mathrm{~m}$ spikes are in the spreader and merger sections (see Fig. 24). The $120 \mathrm{~m}$ long linac section cannot be distinguished, squeezed between spreader and merger lines, it has betatron function values $\beta_{x}=\beta_{y}=120 \mathrm{~m}$ at both ends (see Fig. 27). The 12 regions between the spikes are along the FFAG2 recirculating loop, betatron functions there increase from (see Tab. 1, p. 6) $\beta_{x} / \beta_{y}=0.51 / 6.61 \mathrm{~m}$ amplitude at $6.622 \mathrm{GeV}$ (leftmost $3.887 \mathrm{~km}$ section on the figure) to $\beta_{x} / \beta_{y}=3.57 / 26.1 \mathrm{~m}$ at $21.164 \mathrm{GeV}$ (rightmost). The right vertical axis is for the dispersion functions; $D_{y}$ is non-zero along short chicane segments only, in the spreader and merger lines, $D_{x}$ is in few centimeter range; the small $D_{x}$ oscillation from $15 \mathrm{~km}$ on is due to a slight mismatch, a very small effect anyway ( $\lesssim 10 \mathrm{~cm}$, see Fig. 28). 
- 5.3 to $6.622 \mathrm{GeV}$ linac and spreader optics

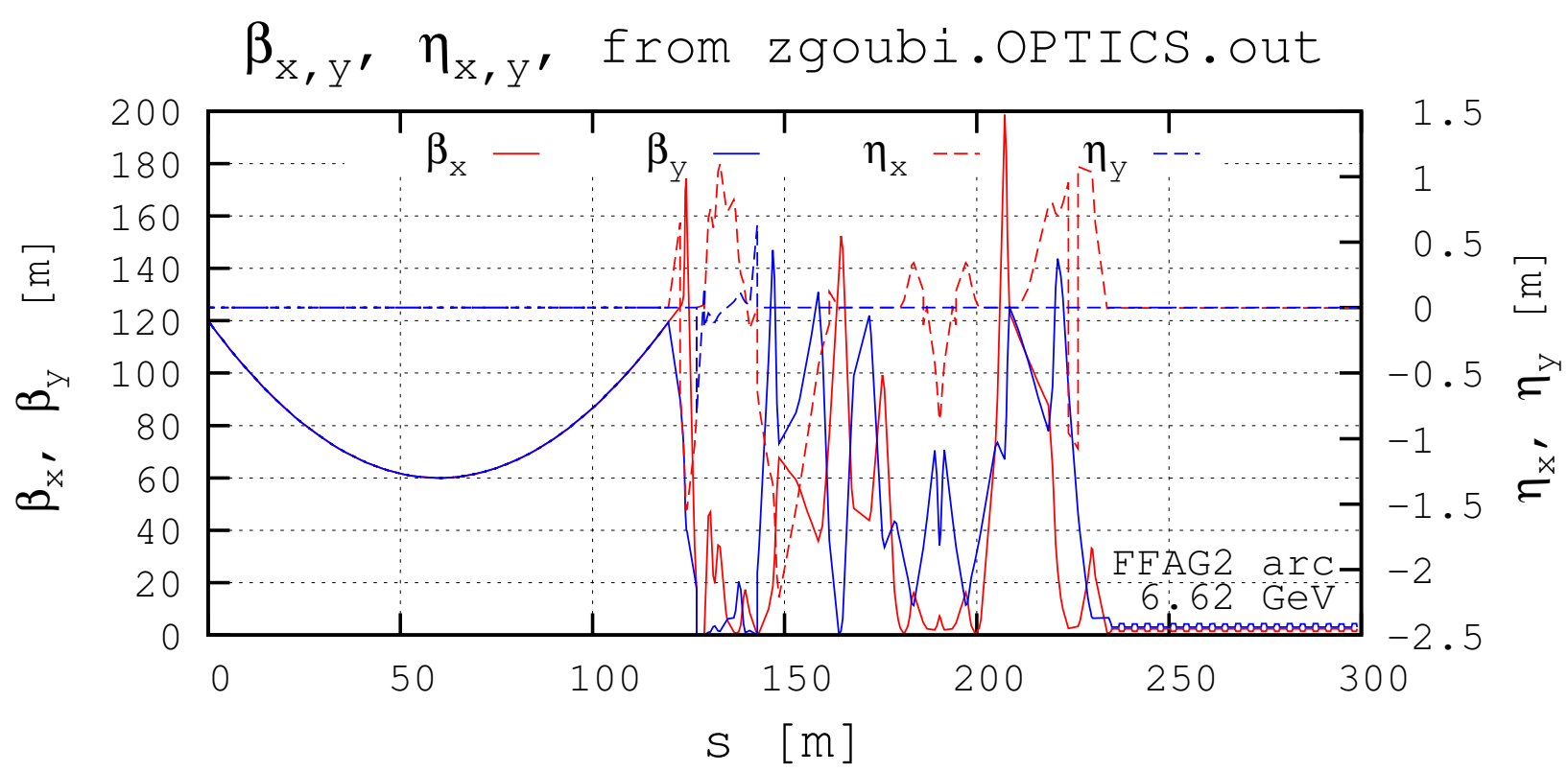

Figure 27: Details of the optical functions (betatron, left axis, and dispersion, right axis), in the case of the $5.3 \rightarrow 6.622 \mathrm{GeV}$ linac energy step. The $\beta_{x}, \beta_{y}$ parabolas at left hand are in the linac (120 m long). The linac is followed by a spreader line which ends up steering the beam on its $6.622 \mathrm{GeV}$ orbit in the FFAG2 loop on the way up. The FFAG2 loop extends in the $s>230 \mathrm{~m}$ region (at right hand), with betatron amplitudes $\beta_{x}, \beta_{y}=5.6,6.6 \mathrm{~m}$ and dispersion function amplitude $-5 \sim+3 \mathrm{~mm}$ (see Fig. 8, p. 5). The latter features $\sim \pm 1.5 \mathrm{~m}$ excursion in the spreader (see Fig. 24, p. 18).

- 19.842 to $21.164 \mathrm{GeV}$ merger-linac-spreader optics

$\beta_{\mathrm{x}, \mathrm{y}}, \eta_{\mathrm{x}, \mathrm{y}}$, from zgoubi.OPTICS.out

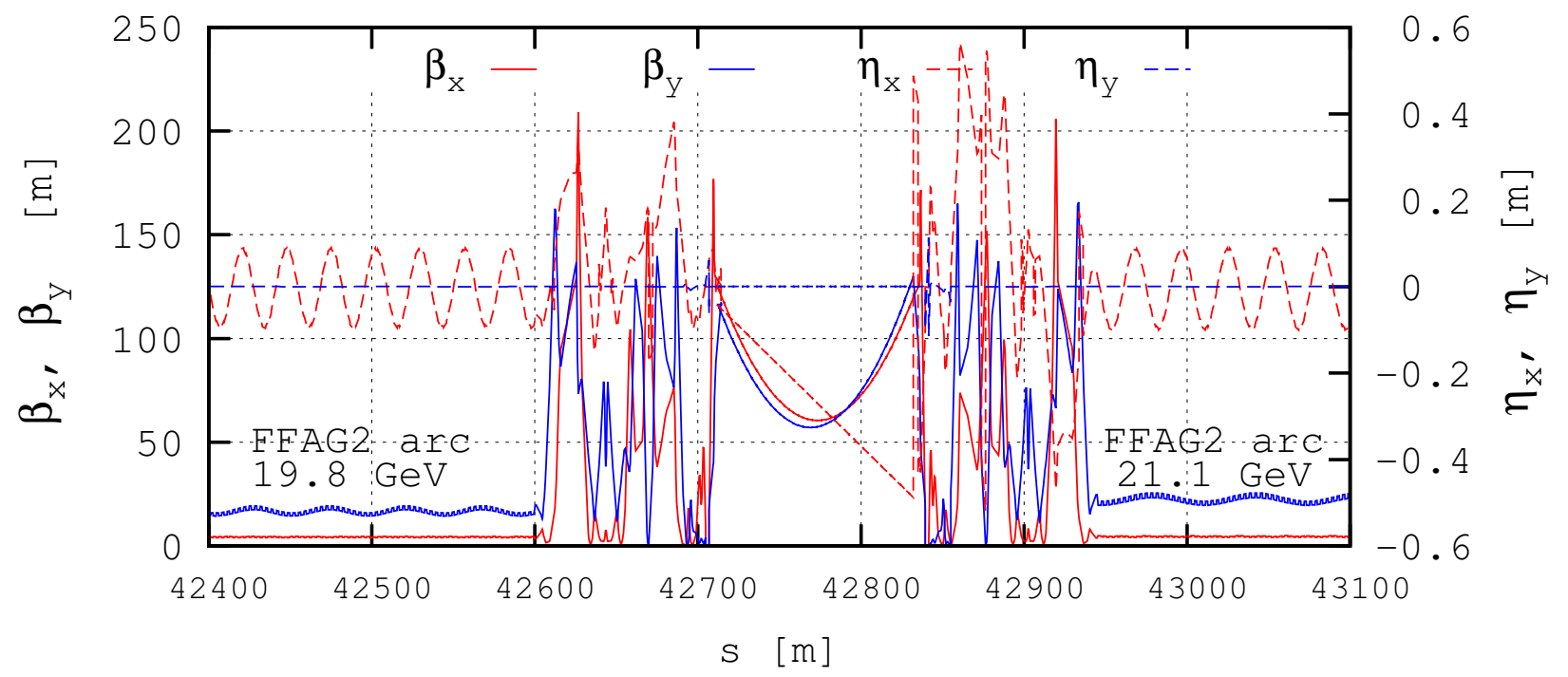

Figure 28: Details of the optical functions (betatron, left axis, and dispersion, right axis), in the $19.842 \rightarrow 21.164 \mathrm{GeV}$ region. The $\beta_{x}, \beta_{y}$ parabolas in the middle region are in the linac. The linac section is preceded by a merger and followed by a spreader line, with, upstream and downstream of the latter, respectively, beam steering from and onto its respectively 19.842 and $21.164 \mathrm{GeV}$ orbit in the FFAG2 loop. The FFAG2 loop extends in the $s<42600 \mathrm{~m}$ and $s>42950 \mathrm{~m}$ regions, with vertical betatron amplitudes $\beta_{y}=20 \mathrm{~m}$ to the left, $\beta_{y}=26 \mathrm{~m}$ to the right (Tab. 1) and, superimposed, an oscillation resulting from cumulated mismatch. The dispersion function has $\pm 10 \mathrm{~cm}$ oscillation in the FFAG loop, due to cumulated upstream mismatch. 


\section{Tracking the ERL}

In this concluding section, the full ERL layout is considered, with optical settings as discussed in Sec. 4 :

$$
\mathrm{ERL}=\underbrace{\begin{array}{c}
\text { Observation point } \\
\text { merger }+\downarrow \text { linac }+ \text { spreader }
\end{array}}_{\text {RHIC IR2 region }}+\text { FFAG2 }
$$

with FFAG2 as in Eq. 10. As pointed earlier, some artifacts and limitations are imposed on the modeling of the ERL, at this stage of its development, as follows.

\section{Artifacts}

- Artificial bunch centroid centering is applied along the ERL (using Zgoubi's "AUTOREF" keyword), as follows : - at exit of any of the 12 merger lines (i.e., at entrance of linac) :

- (i) horizontal $\left(x, x^{\prime}\right)$ and vertical $\left(y, y^{\prime}\right)$ bunch centering on zero (a substitute to beam steering onto linac optical axis),

- (ii) bunch centering on design momentum (this stands for artificial compensation of SR loss in the upstream FFAG arc and merger line),

- (iii) time centering so that at any stage in the acceleration-deceleration cycle bunches will enter the linac centered on the RF crest,

- at exit of any of the 12 spreader lines : bunch centering on current FFAG orbit (a substitute to beam steering), centering on design momentum (this stands for artificial compensation of SR loss in the spreader),

- at entrance to each of the five LSS (i.e., going from arc to straight) : horizontal (x,x') and vertical (y,y') bunch centering on zero (this cancels (i) SR induced orbit in the arcs, and (ii) induced orbit by the DS section).

- Limitations in the model in relation with these artifacts and with other approximations which they entail, include :

- SR is switched off in all (and there only) spreader and merger lines except in the top energy spreader and merger lines, $21.164 \mathrm{GeV}$. As a consequence, except for the latter, their contributions to SR induced energy losses and related beam and spin dynamics effects are not accounted for,

- same for spin tracking, switched off in all (and only there) 6.622 to $19.842 \mathrm{GeV}$ spreader and merger lines.

\subsection{Way up, 6.622 to $21.164 \mathrm{GeV}$}

\subsubsection{Beam ellipses at linac ends}

Correct behavior of the tracking is first assessed at linac ends : one hundred particles evenly distributed on paraxial invariant with $\beta=120 \mathrm{~m}, \alpha= \pm 1$ (both horizontal and vertical) are launched at linac entrance with $\mathrm{E}=5.3 \mathrm{GeV}$, for a 12 linac-pass tracking up to $21.164 \mathrm{GeV}$. Betatron damping has been inhibited in this case (Chambers matrices, Eq. 7, are normalized to determinant 1).

It results from the tracking that beam ellipse parameters remain $\beta=120 \mathrm{~m}, \alpha= \pm 1$ at a few $\%$ level at both linac ends, both planes, all the way from 5.3 to $21.164 \mathrm{GeV}$, see Fig. 29.

\subsubsection{0-particle bunches at linac ends}

A 5000-particle bunch is tracked here. We show that transverse and longitudinal bunch emittances, as observed at linac ends, behave in a reasonable manner - details require further investigation.

Initial bunch emittances, at $5.3 \mathrm{GeV}$, are $23 \mu \mathrm{m}$ transverse, zero longitudinal (both length and $\mathrm{dE} / \mathrm{E}$ zero). Linac damping is accounted for, SR as well.

Results are displayed in Figs. 30, Figs. 31. 

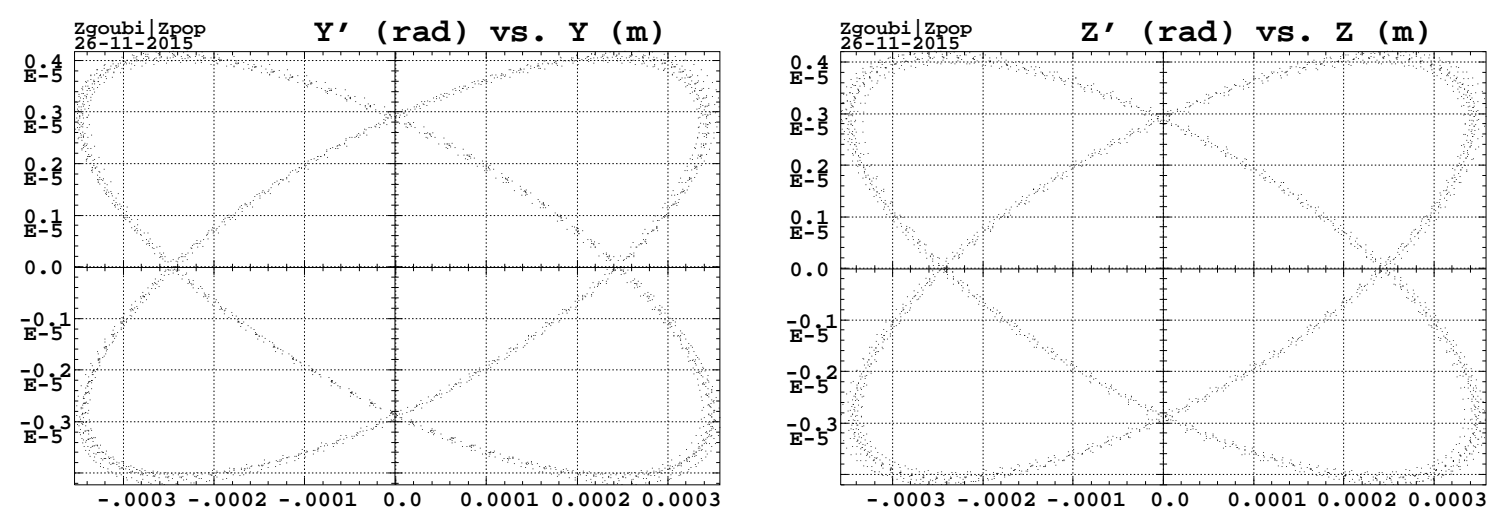

Figure 29: The figure shows a superimposition of 12, 100-particle bunches, at linac entrance (each 100 particle set is spread on a converging ellipse, all 12 ellipses do superimpose) and the same bunches at linac exit (each 100 particle set is spread on a diverging ellipse, all 12 ellipses do superimpose).

$\bullet\left(x, x^{\prime}\right)$, AT LINAC ENDS

5.300 AND 6.622 GeV

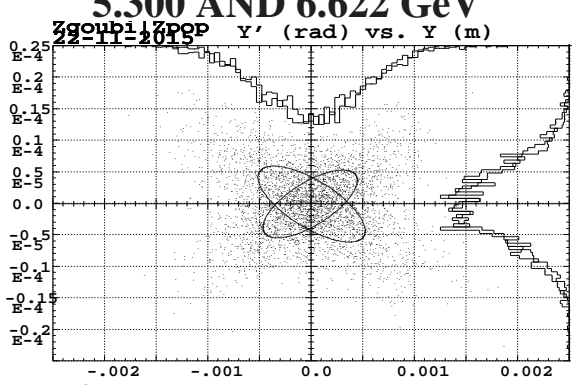

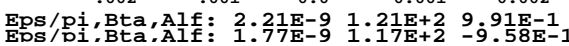

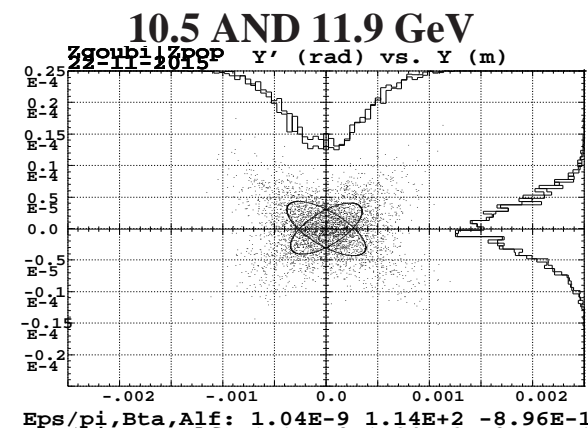

19.8 AND $21.164 \mathrm{GeV}$

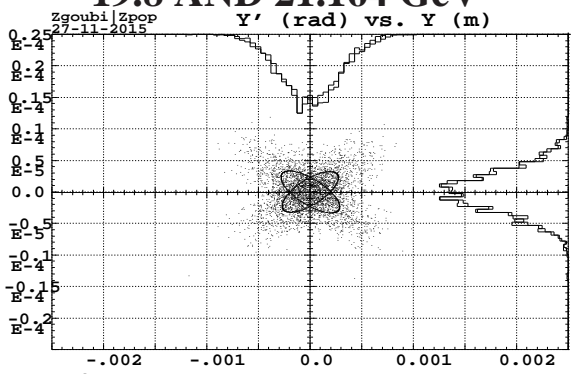

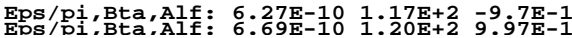

• $\left(\mathrm{y}, \mathrm{y}^{\prime}\right)$, AT LINAC ENDS
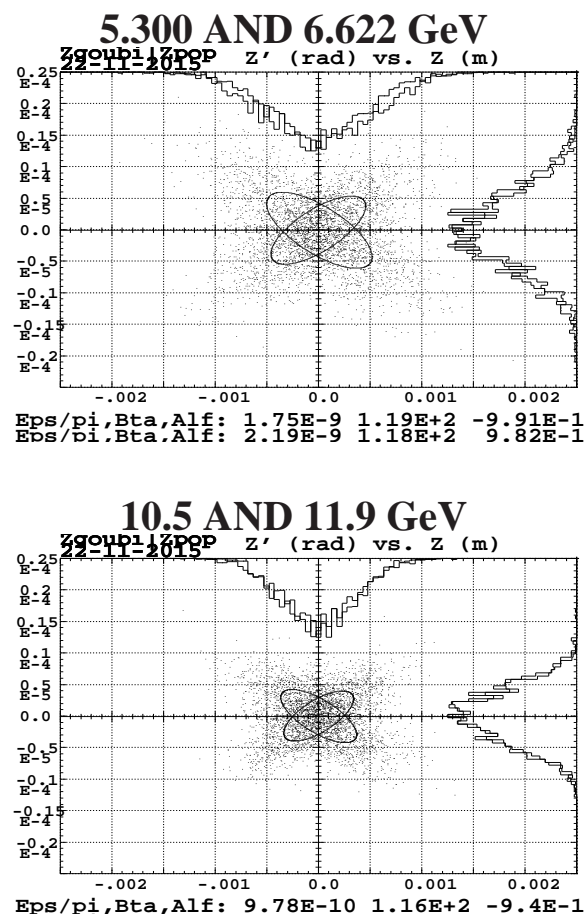

Eps/pi, Bta, Alf:

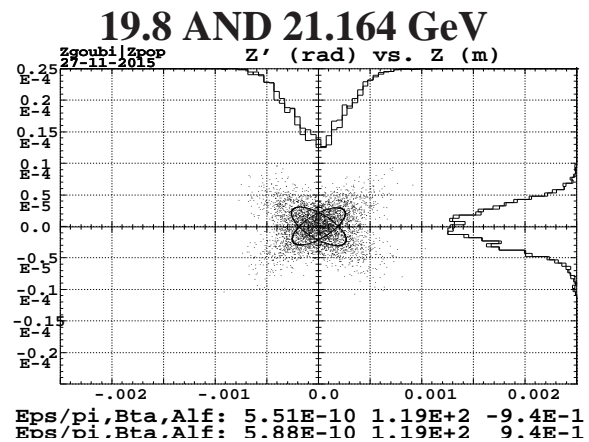

Figure 30: Bunch transverse emittances (non normalized) and $\beta_{x}, \alpha_{x}, \beta_{y}, \alpha_{y}$ parameters are given at the bottom of the plot for each energy. These parameters appear to be well preserved, with $\beta \gamma$ betatron damping as expected (i.e., same normalized emittances at linac entrance and exit). 


\section{- (time, kinetic energy), AT LINAC EXIT (initial bunch length and dE/E null)}

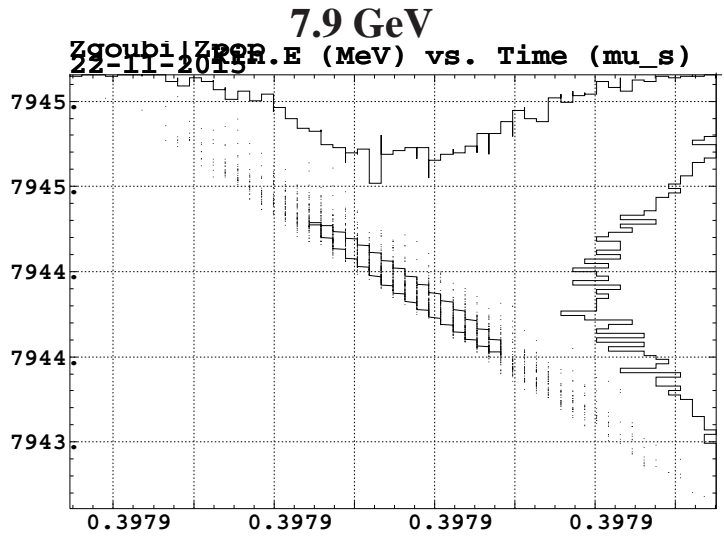

Ens/di. Bta.Alf: $1.77 \mathrm{E}-8 \quad 3.18 \mathrm{E}-6 \quad 5.11 \mathrm{E}+0$

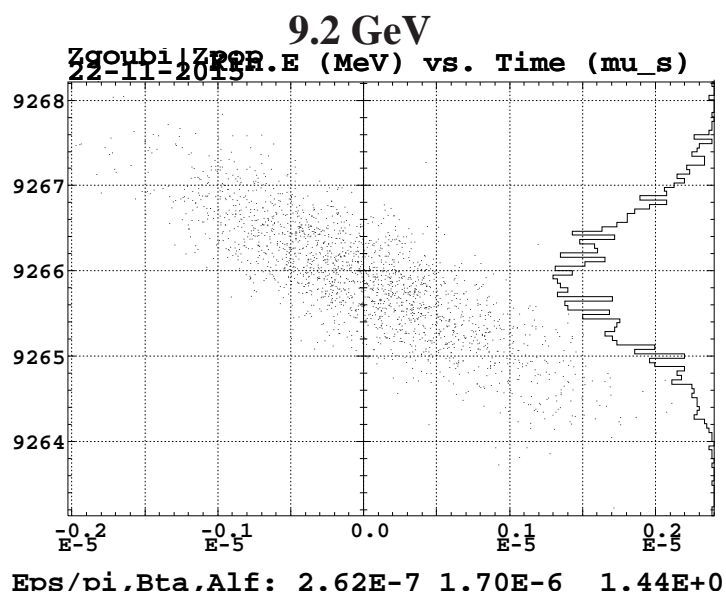

$10.5 \mathrm{GeV}$

Zgoupi=2Jpgr.E (Mev) vs. Time (mu_s)

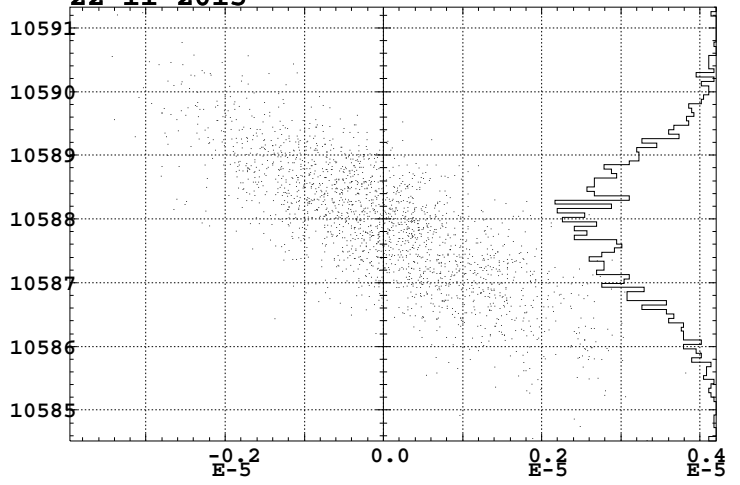

Eos/di.Bta.Alf: $7.40 \mathrm{E}-7 \quad 1.88 \mathrm{E}-6 \quad 1.22 \mathrm{E}+0$

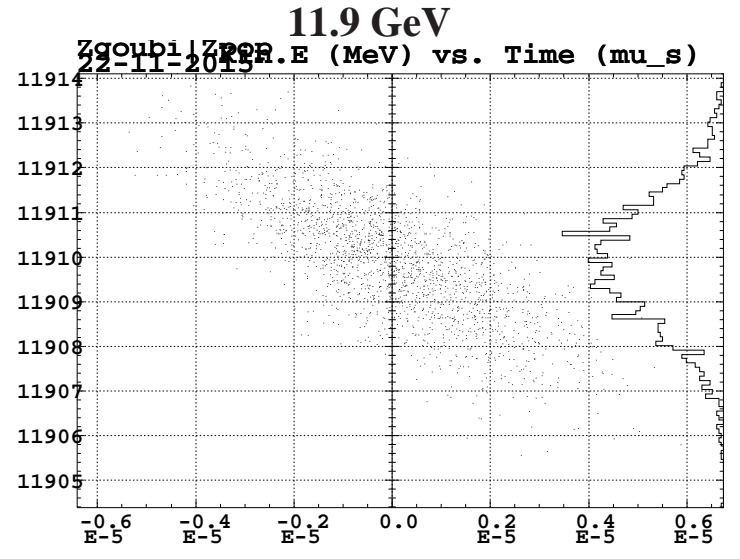

Eos/di. Bta. Alf : $1.58 \mathrm{E}-6 \quad 2.02 \mathrm{E}-6 \quad 1.15 \mathrm{E}+0$

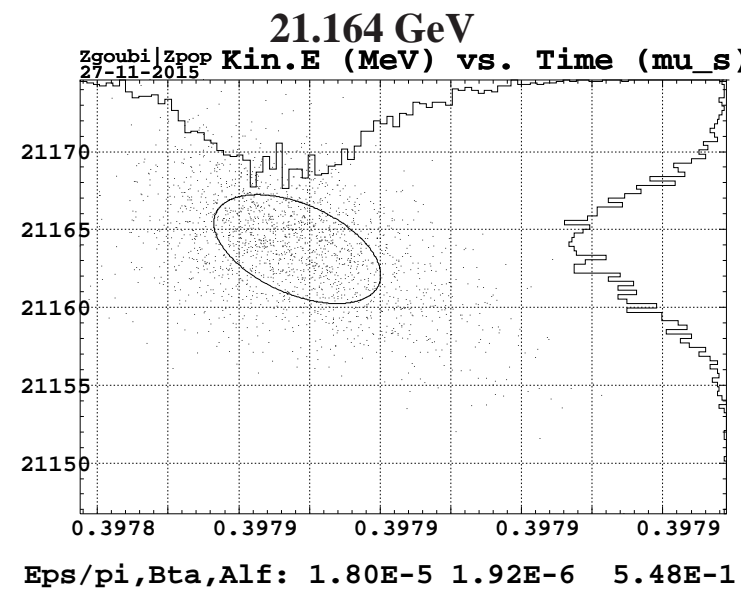

21.164 GeV centered

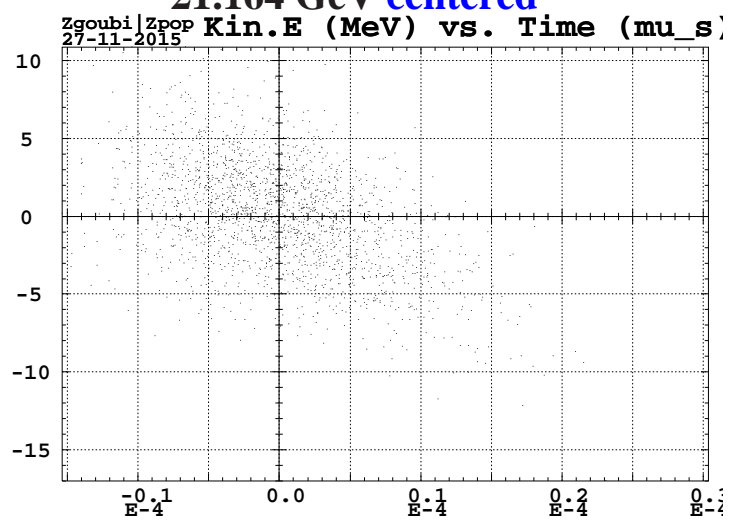

Eps/pi, Bta, Alf: $1.79 \mathrm{E}-5 \quad 1.91 \mathrm{E}-6 \quad 5.41 \mathrm{E}-1$

Figure 31: Bunch longitudinal rms emittance (in $\mu \mathrm{eV} . \mathrm{s}$ ) is given at the bottom of the plot for each energy. It appears to behave reasonably well (simulation wise) all the way from 5.3 to $21.16 \mathrm{GeV}$. Initially zero (at $5.3 \mathrm{GeV}$ ), $\epsilon_{l}$ remains small at top energy (bottom right plot) - growth mechanisms include SR, details require further investigation. 


\subsection{Up-down cycle in the FFAG2 stage ERL}

\subsubsection{Linac damping off and SR off}

In order to ensure that input data files for the 24 linac passes end-to-end tracking are set up correctly, a preliminary up-down cycle is performed with linac damping off and synchrotron radiation off. A 2000 particle bunch is tracked, initial bunch emittance is

$$
\beta_{x, \text { norm }}=\beta_{y, \text { norm. }}=23 \pi \mu \mathrm{m}, \quad \sigma_{l}=0, \quad \sigma_{E}=0
$$

Transverse emittances are expected to be preserved, and longitudinal beam size growth is expected to be commensurate with SR induced growth observed in the case of the 6-arc model, Sec. 2.4.

Tracking results are displayed in Figs. 32, 33. In Fig. 32, a particle is represented by an empty box marker, it can be seen that at each energy the 2000 boxes superimpose perfectly - at that scale. Fig. 33 shows phase space details at the end of the acceleration-deceleration cycle, back to $5.3 \mathrm{GeV}$. This tracking demonstrates the preservation of the orbits and of the transverse emittances, and small longitudinal emittance growth, over a complete $5.3 \stackrel{\text { up }}{\rightarrow} 21.164 \stackrel{\text { down }}{\rightarrow} 5.3 \mathrm{GeV}$ cycle.

Note that no symplecticity issue is expected: tracking distance here is very short compared to routinely 100 thousands of turns tracking trials performed for proton polarization studies in RHIC, using similar integration step size, and non-linear optics.

\subsubsection{Linac damping and SR set}

A important aspect at this stage : there has been no optimization effort regarding bunch transmission, this is out of the scope of the present stage which concerns the setting up of the data and data files for end-to-end simulation studies. Instead, bunch transmission studies and optimization are planned to lean on the tools so developed, amongst others.

This said, tracking is performed here with synchrotron radiation and with unnormalized Chambers matrices (i.e., betatron damping accounted for). The results are displayed and commented in Figs. 34, 35.

Fig. 34 shows that the bunch undergoes noticeable (at the scale of the figure) energy spreading beyond pass $18 \sim 19$ (markers no longer superimpose).

Transverse emittance growth observed in Fig. 35 requires further investigation, this is part of the end-to-end simulation work planned. The large extent and sine-like distortion of the bunch in longitudinal phase space at the final energy after deceleration, $5.3 \mathrm{GeV}$, can be seen in the bottom plot in Fig. 35. It can be compared with the SR free case, bottom plot in Fig. 33.

Fig. 36 shows the evolution of SR energy loss over 23 recirculations from 5.3 to $21.1 \mathrm{GeV}$ and back to $5.3 \mathrm{GeV}$. For the record, because their design is not optimized at the time that these simulations are performed, $\mathrm{SR}$ is maintained off in all spreader and merger lines.

Note that, as part of the artifacts discussed in page 22, bunches always present themselves on phase with RF crest at linac entrance. 


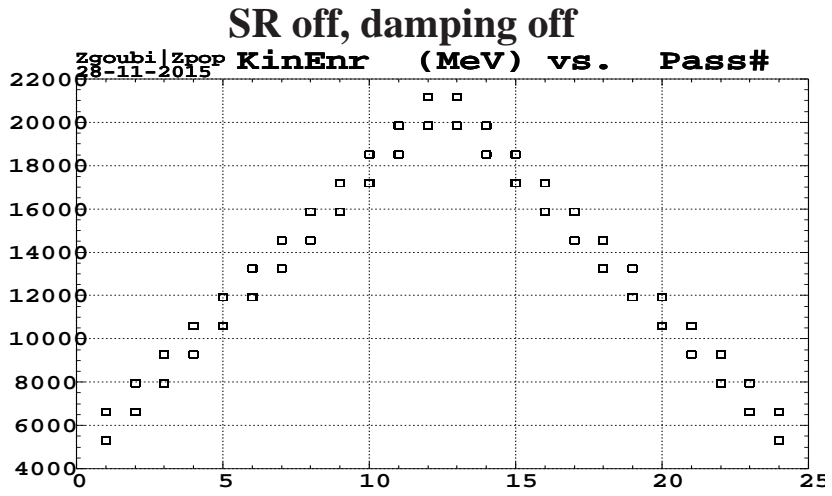

Figure 32: Average kinetic energy of a 2000-particle bunch, at entrance and exit of the linac (hence two markers per pass, aligned vertically), as a function of pass number (each particle is represented by an empty box). The bunch remains well confined (ech box is a superimposition of 2000) from $5.3 \mathrm{GeV}$ injection up to $21.164 \mathrm{GeV}$ and back down to $5.3 \mathrm{GeV}$.

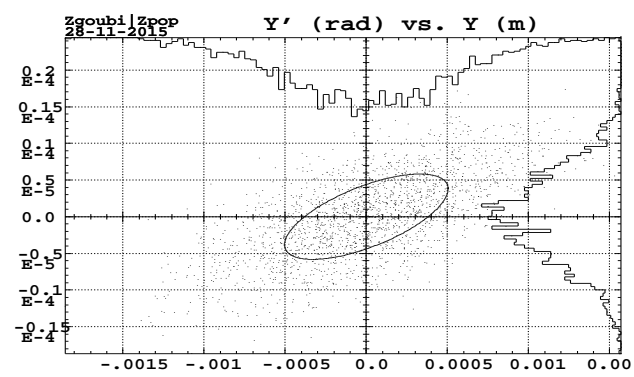

Eps/pi, Bta, Alf: 2.2155E-09 1.1473E+02 -8.6682E-01
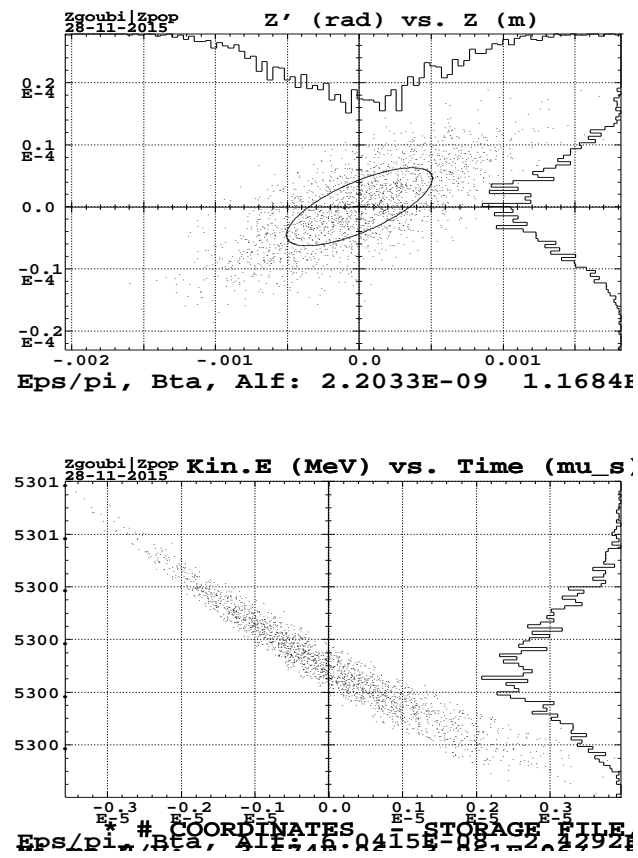

Figure 33: Phase spaces back to $5.3 \mathrm{GeV}$, horizontal (top), vertical (middle) and longitudinal (bottom). The former two feature a preserved $23 \mu \mathrm{m}$ normalized emittance, the latter shows very small final longitudinal emittance.

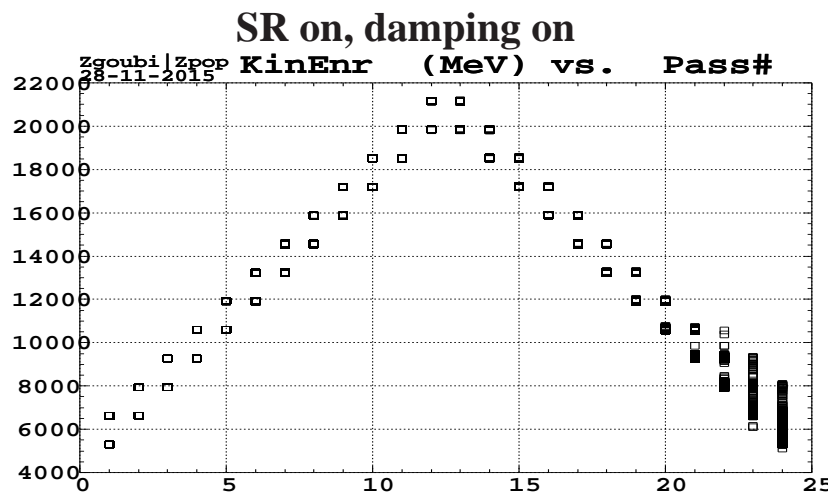

Figure 34: Average kinetic energy of the 2000-particle bunch, at entrance and exit of the linac (hence two markers per pass), as a function of pass number (each particle is represented by an empty box). The bunch appears to undergo serious energy spreading from pass $12 \sim 13$ on to final $5.3 \mathrm{GeV}$.
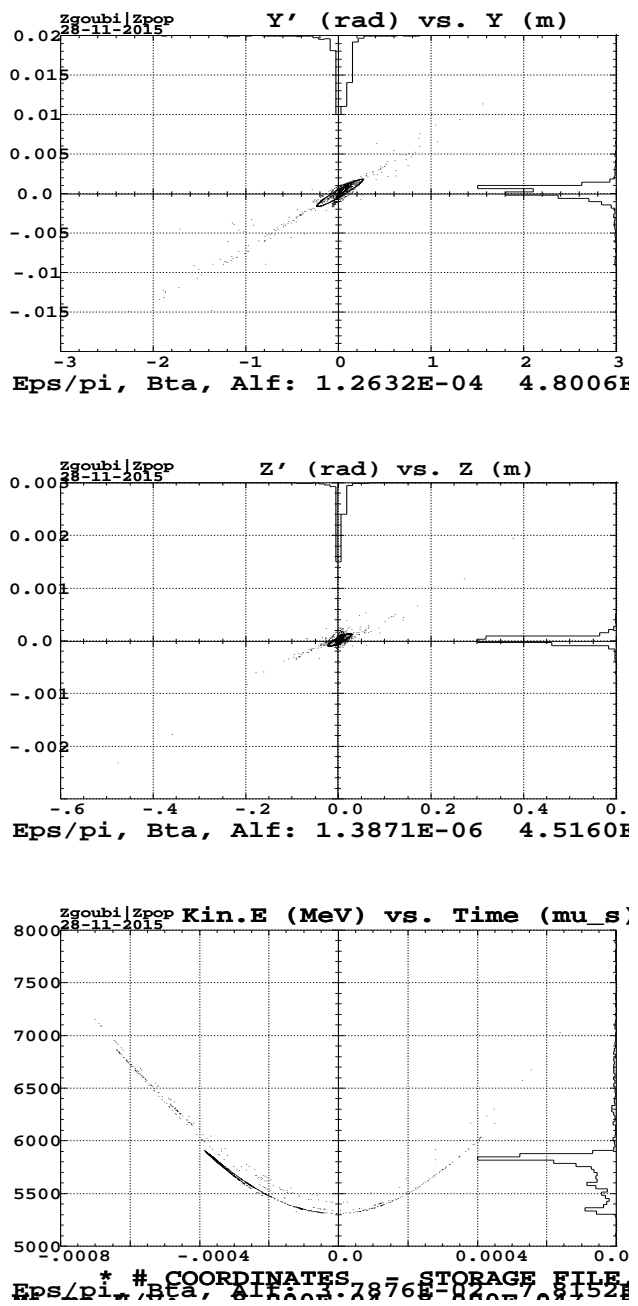

Figure 35: Phase spaces at $5.3 \mathrm{GeV}$ after 24 recirculations, horizontal (top), vertical (middle) and longitudinal (bottom). The effects of SR are substantial. 
SR ENERGY LOSS IN FFAG2

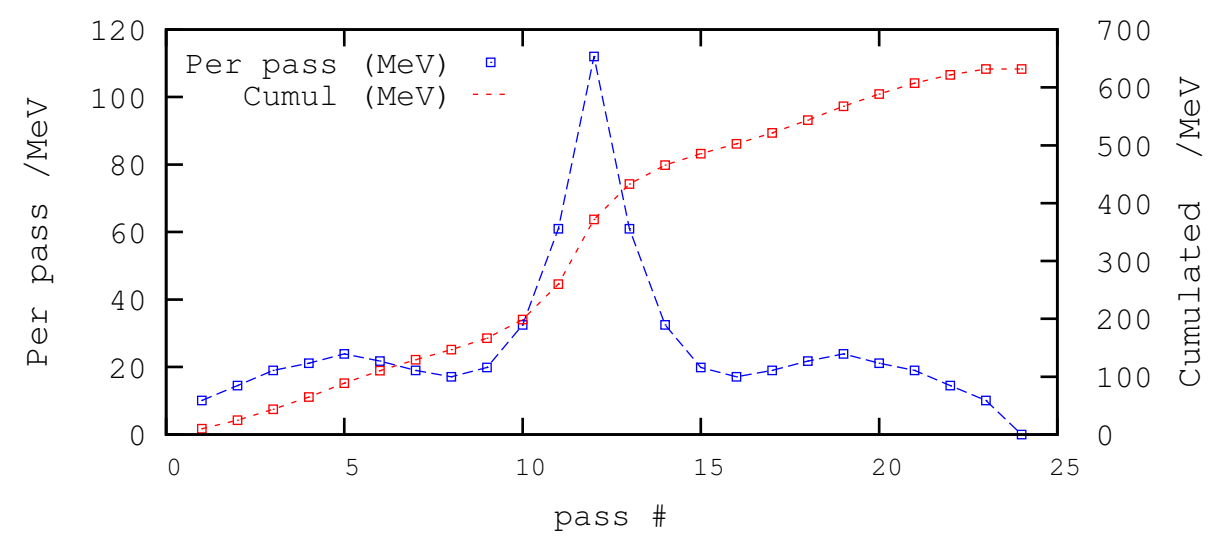

Figure 36: Evolution of SR energy loss over 23 recirculations from 5.3 to $21.1 \mathrm{GeV}$ and back to $5.3 \mathrm{GeV}$. There are various causes to the non-symmetry of the "per pass" curve with respect to pass number 12, this is to be investigated further.

\subsection{Polarization}

The polarization state out of these simulation data file preparations, for a 5000 particle bunch at top energy after acceleration from 5.3 to $21.164 \mathrm{GeV}$, is displayed in Fig. 37, in both SR off and SR on cases.

\section{- Bunch polarization at collision energy, 21.164 GeV •}

\section{- SR off :}
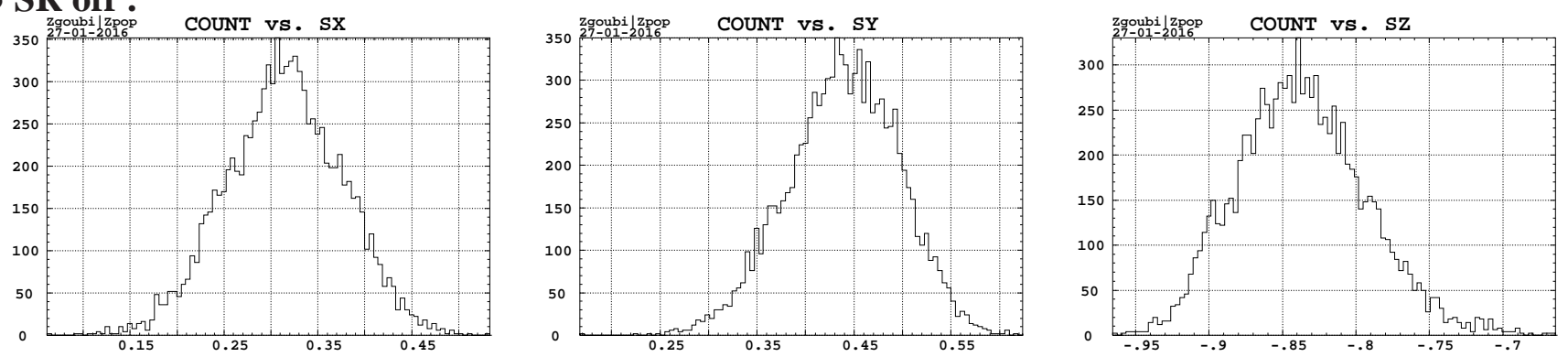

\section{- SR on}
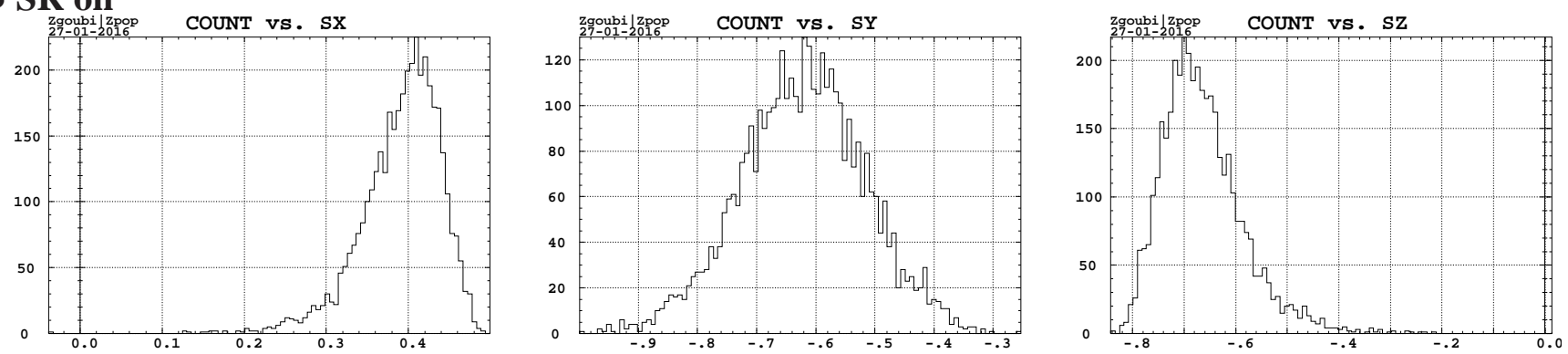

Figure 37: Bunch polarization states (5000 particles) at top energy, SR off (top row) and SR on (bottom row). 


\section{Prospects}

Preliminary developments of a computer model of eRHIC ERL have been discussed, the modeling uses the raytracing code Zgoubi, it is based in the present report on the June 2015 optics version of eRHIC. This preliminary computer model includes various approximations and relies on artifacts at various locations along the layout, in various aspects as beam steering and alignment.

However this model is under continuous development,

- it is updated as eRHIC parameters evolve (e.g., linac energy, FFAG rings optics),

- artifacts and limitations will be removed as the information (design of the various optical modules, details of bunch manipulations, etc.) becomes available,

This model allows preliminary and qualitative, yet thorough in many aspects, investigations regarding methods for, and outcomes of, the simulation of end-to-end polarized electron bunch transport and energy recovery in eRHIC ERL.

The development of this model in Zgoubi will improve with time in the details of lattice optics and bunch dynamics, and will accompany the evolution of eRHIC layout. Further computer code developments aimed at refining the polarized electron bunch transport simulations include main potential collective effects as BBU and other halo generation mechanisms. 


\section{Appendix}

\section{A FFAG2 arc cell in Zgoubi}

The deviation in the cell is $\theta_{\text {cell }}=\theta_{F}+\theta_{D}=8.731028 \mathrm{mrad}$, whatever the energy (the sum of the two quantities 1.6564835E-03, 2.7090305E-03, below, times 2). The FFAG2 loop in the ERL is comprised of 6 arcs formed with a series of 102 such cells. (On the other hand, 120 cells are necessary to close the circle in the case of the simple 6-arc model used in Sec. 2.4.)

Note also that the average curvature radius, $<\rho>=\left(\rho_{F} \theta_{F}+\rho_{D} \theta_{D}\right) / \theta_{\text {cell }} \approx 341 \mathrm{~m}$, depends only marginally upon energy, since the dispersion function is small (arc length does not change much with energy). This quantity is used in estimating spin diffusion using Eq. 5.

The radial shift of the BD (defocusing) and QF (focusing) magnets (Figs. 2, 3) is accounted for in the present simulations via a dipole component and no shift at all (an arbitrary choice, proceeding the other way would be as valid). Namely, the dipole component in the combined function BD magnet data below, $B_{D}=0.0293364 \mathrm{kG}$, is equivalent to a radial shift of a $G_{D}=-0.5225857 \mathrm{~T} / \mathrm{m}$ gradient quadrupole by $\Delta x_{D}=B_{D} / G_{D}=-5.61 \mathrm{~mm}$; the dipole component in the combined function QF magnet, $B_{F}=0.0293343 \mathrm{kG}$, is equivalent to a radial shift of a $G_{F}=+0.3728876 \mathrm{~T} / \mathrm{m}$ gradient quadrupole by $\Delta x_{F}=B_{F} / G_{F}=+7.87 \mathrm{~mm}$, in the opposite direction, hence a $13.48 \mathrm{~mm}$ inter-axis distance between the two quadrupole-equivalents (prior to their respective $\theta_{D}$ and $\theta_{F}$ tilting).

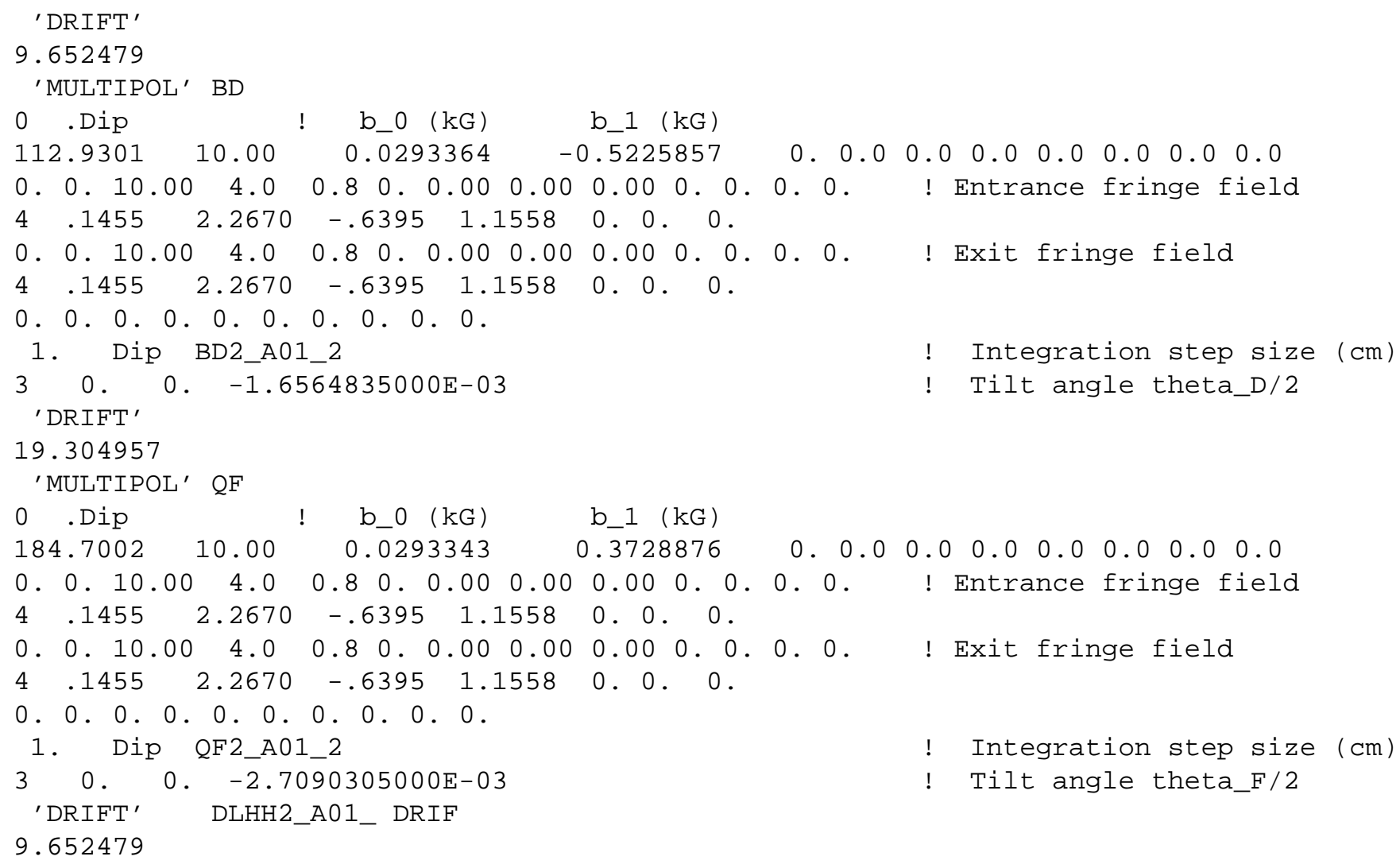

\section{B A linac cavity in Zgoubi}

The length datum, $L=1.7749 \mathrm{~m}$ here, is not used in the transport matrix, it is given though, for the purpose of time of flight updating.

\footnotetext{
'DRIFT' CAV_UP

1.41994249041e2
} 


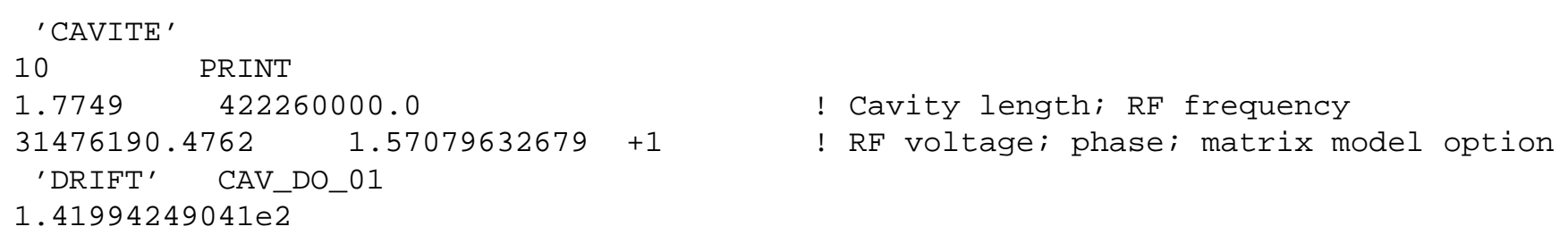

\section{References}

[1] E.C. Aschenauer et al., "eRHIC Design Study, Electron-Ion Collider at BNL”, arXiv:1409.1633, Sept. 2014.

[2] F. Méot, The ray-tracing code Zgoubi - Status, NIM A 767 (2014) 112125.

[3] F. Méot, Zgoubi users’ guide, http://www.osti.gov/scitech/biblio/1062013

[4] F. Méot et al., Tracking studies in eRHIC energy-recovery recirculator, eRHIC Tech. Note 45 (July 2015).

[5] D. Trbojevic, FFAG Lattice Design of eRHIC and LHeC, EIC'14 workshop, http://appora.fnal.gov/pls/eic14/agenda.full.

[6] F. Méot, Simulation of radiation damping in rings, using stepwise ray-tracing methods, 2015 JINST 10 T06006 (June 2015).

[7] G. Leleux et al., Synchrotron radiation perturbation in transport lines, Part. Acc. Conf., San Francisco, May 6-9, 1991.

[8] V. Ptitsyn, Electron Polarization Dynamics in eRHIC, EIC 14 workshop, JLab, 17-21/03/2014, http://appora.fnal.gov/pls/eic14/agenda.full.

[9] J. Rosenzweig, L. Serafini, Transverse particle motion in radio-frequency linear accelerators, Phys. Rev., E49 (1994) 1599.

[10] https://oraweb.cern.ch/pls/hhh/code_website.disp_code?code_name=BETA

[11] F. Méot, Focusing in the LINAC (Phase 1), CEA/LNS/GT/ME/92-05, Saclay (1992). 